\title{
Asymmetric jet production in the active galactic nucleus of NGC 1052^
}

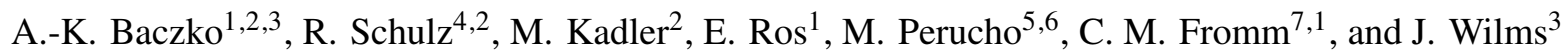 \\ 1 Max-Planck-Institut für Radioastronomie, Auf dem Hügel 69, 53121 Bonn, Germany \\ e-mail: baczko@mpifr-bonn.mpg.de \\ 2 Institut für Th. Physik und Astrophysik, Univ. Würzburg, Emil-Fischer-Str. 31, 97074 Würzburg, Germany \\ 3 Dr. Remeis-Sternwarte \& ECAP, Univ. Erlangen-Nürnberg, Sternwartstr. 7, 96049 Bamberg, Germany \\ 4 Netherlands Institute for Radio Astronomy (ASTRON), PO Box 2, 7990 AA Dwingeloo, The Netherlands \\ 5 Observatori Astronòmic, Univ. de València, C/ Catedrático José Beltrán no. 2, 46980 Paterna, València, Spain \\ 6 Dep. d'Astronomia i Astrofísica, Univ. de València, C/ Dr. Moliner 50, 46100 Burjassot, València, Spain \\ 7 Institut für Theoretische Physik, Goethe Universität, Max-von-Laue-Str. 1, 60438 Frankfurt, Germany
}

Received 11 July 2018 / Accepted 21 December 2018

\begin{abstract}
Context. Few active galactic nuclei (AGN) reveal double-sided jet systems. However, these systems are crucial to understand basic physical properties of extragalactic jets.

Aims. We address the questions whether jets in AGN are symmetric in nature, how well they are collimated on small scales, and how they evolve with time.

Methods. We monitored the sub-parsec scale morphology of NGC 1052 with the Very Long Baseline Array at $43 \mathrm{GHz}$ from 2005 to 2009.

Results. A detailed study of 29 epochs show a remarkable asymmetry between both jets. A kinematic analysis of the outflows reveals higher apparent velocities for the eastern (approaching) jet as compared to the western (receding) jet, i.e., $\beta_{\text {ej }}=0.529 \pm 0.038$ and $\beta_{\mathrm{wj}}=0.343 \pm 0.037$, respectively. Contradictory to previous studies, we find higher flux densities for the western jet as compared to the eastern. The distribution of brightness temperature and jet width features well-collimated jets up to 1 mas distance to the dynamic center and a nearly conical outflow further outward. By combining flux density ratios and velocities of the jet flows, we were unable to find a combination of intrinsic velocities and inclination angles of the jets that is consistent for all four years of observation; this contradicts findings for symmetrically evolving jets. Spectral index maps between quasi-simultaneous $22 \mathrm{GHz}$ and $43 \mathrm{GHz}$ observations support the existence of an optically thick absorber covering the innermost $\simeq 1.6$ mas around the $43 \mathrm{GHz}$ central feature and an optically thin jet emission with a spectral index of $\leq-1$.

Conclusions. Our results fit into a picture in which we expect larger internal energy and/or magnetic flux in the western jet and higher kinetic energy in the eastern jet. Previous observations at lower frequencies have found slower velocities of the moving jet features as compared to this work. Considering the different velocities in different areas, we suggest a spine-sheath structure with a faster inner layer and slower outer layer.
\end{abstract}

Key words. galaxies: active - galaxies: jets - methods: observational - galaxies: kinematics and dynamics - galaxies: nuclei

\section{Introduction}

Relativistic jets observed in radio-loud active galactic nuclei (AGN) are intensively studied, yet key questions remain regarding the formation, collimation, and evolution especially on (sub-) parsec scales. Theoretical models by Blandford \& Königl (1979) or Königl (1981) describe narrow, conical evolving jets with a constant velocity. In addition, most models assume two symmetric jets ejected in opposite directions from the central engine. Numerical simulations, however, show that asymmetric jets can be produced (see, e.g., Fendt \& Sheikhnezami 2013). Observationally, it is difficult to find a suitable AGN with (1) both jets prominently visible and sufficiently strong at high frequencies, (2) the jets oriented in the plane of the sky to avoid effects of the differential Doppler boosting, and (3) a close distance in order to reach small spatial scales.

* The FITS files of the Q-Band data are only available at the CDS via anonymous ftp to cdsarc.u-strasbg. fr (130.79.128.5) or via http://cdsarc.u-strasbg.fr/viz-bin/qcat?]/A+A/623/A27
The radio galaxy NGC 1052 fulfills these requirements. This galaxy hosts a supermassive black hole $(\mathrm{SMBH})$ with a mass on the order of $\approx 10^{8.2} M_{\odot}$ (Woo \& Urry 2002). Because of its optical spectrum, this radio galaxy is classified as a low-ionization nuclear emission-line region object (Mayall 1939; Fosbury et al. 1978; Ho et al. 1997).

NGC 1052 is located at a redshift of $z=0.005037$ (assuming $v_{\text {sys }}=1507 \mathrm{~km} \mathrm{~s}^{-1}$; Jensen et al. 2003). For nearby objects such as NGC 1052 distance estimates based on recession velocities are biased by peculiar velocities of the galaxies. We use, therefore, the redshift-independent distance of $D=19.23 \pm$ $0.14 \mathrm{Mpc}$ (Tully et al. 2013). This results in a linear scale of $0.093 \mathrm{pc} \mathrm{mas}^{-1}$.

At parsec-scales NGC 1052 exhibits two jets, which emanate from the center and show an emission gap at centimeter wavelengths that becomes smaller toward higher frequencies. This effect is from free-free absorption (FFA) caused by a geometrically thick structure perpendicular to the jets with an optical depth $\tau_{1 \mathrm{GHz}} \sim$ 300-1000 (Kameno et al. 
2001; Sawada-Satoh et al. 2008) and a column density of $10^{22} \mathrm{~cm}^{-2}-10^{24} \mathrm{~cm}^{-2}$ (Kameno et al. 2001; Vermeulen et al. 2003; Kadler et al. 2004a). This circumnuclear torus covers larger parts of the western, receding jet. Its absorbing effect becomes smaller toward higher frequencies and has close to no impact at $43 \mathrm{GHz}$. Several authors reported on $\mathrm{H}_{2} \mathrm{O}$ maser emission, which is most likely associated with the surrounding torus (Claussen et al. 1998; Kameno et al. 2005; Sawada-Satoh et al. 2008). Based on multifrequency observations performed in 2000, Sawada-Satoh et al. (2008) identified the $\mathrm{H}_{2} \mathrm{O}$ masers projected on the eastern and western jet components as B and C3, respectively. The mean speed of features in both jets have been derived as $v / c=\beta \leqslant 0.23$ (Vermeulen et al. 2003; Böck 2012; Lister et al. 2013).

Baczko et al. (2016a) presented the first detection of the twin jets in NGC 1052 at $86 \mathrm{GHz}$. These results show a compact, bright central feature and two faint plasma streams. The central feature can be interpreted as blended emission from both jet cores. Therefore, this central feature pinpoints the location of the central engine. Assuming basic synchrotron theory, this sets boundaries to the strength of the magnetic field at $1 R_{\mathrm{S}}$ : $200 \mathrm{G}<B<8 \times 10^{4} \mathrm{G}$.

In this paper, we investigate this exceptional source further at $43 \mathrm{GHz}$ and $22 \mathrm{GHz}$ based on 29 observations over four years with the Very Long Baseline Array (VLBA). In Sect. 2 we introduce the observations. Section 3 gives an overview of the methods used to derive the physical quantities. In Sect. 4, we discuss the different aspects of our analysis, and Sect. 5 summarizes our results and gives an outlook.

\section{Observation and data reduction}

\subsection{Imaging}

NGC 1052 was observed with the VLBA over 29 epochs (full track) between 2005 March and 2009 April quasisimultaneously at $22 \mathrm{GHz}$ and $43 \mathrm{GHz}$. The data were recorded with eight sub-bands with a bandwidth of $8 \mathrm{MHz}$ and data rate of $256 \mathrm{Mbps}$. The fringe finding calibrators were III Zw 2 and B0420-014. The correlation was performed at the National Radio Astronomy Observatory (NRAO) at the Array Operation Center of the VLBA in Socorro, New Mexico, USA.

A priori amplitude calibration was performed using antenna gain curves and measured system temperatures at all stations. Solutions have been found for the relative delays between observing sub-bands (manual phase-cal) and a fringe search has been conducted to obtain residual delays and delay rates (fringe process). These tasks were performed in AIPS. After averaging over frequencies within each sub-band data were exported from AIPS to perform hybrid mapping in DIFMAP (Shepherd et al. 1994). Several iterations of the CLEAN algorithm and phase and amplitude self-calibration loops yielded the final images used in our analysis. Table 1 lists the final $43 \mathrm{GHz}$ image parameters.

\subsection{Gaussian model fitting}

To parameterize the image properties, we fitted two-dimensional Gaussian functions to the visibility data in DIFMAP. This was performed independently for each observation until satisfactory models for all epochs were obtained. Hence, the model is stable and reduces the noise in the image as much as possible. The parameters of all model fitting procedures are listed in the Appendix (Tables A.2-A.6). The parameters total flux, distance, and major axis were set free in the model. The axial ratio for most jet components was fixed to unity to get the most satisfactory model, whereas it was left as a free parameter for a large portion of the core components. For component identification, images were aligned on the central bright feature that is observable in almost all epochs. It is located at the map peak, and, therefore, at the map origin. This approach differs from that used by Böck (2012), who aligned the maps at $15 \mathrm{GHz}, 22 \mathrm{GHz}$, and $43 \mathrm{GHz}$ on the center of the emission gap that is dominant at frequencies of $22 \mathrm{GHz}$ and below. As we see no indication of strong absorption effects at $43 \mathrm{GHz}$, the central bright feature is assumed to pinpoint the position of the central SMBH and thus remains constant over time. The models were iterated, meaning various combinations of number, position, and shape of Gaussian components were tested to find consistency between adjacent epochs. Three datasets were excluded from the analysis (BR099B, BR099H, and BR120G) because of poor data quality. For the amplitude values we assumed an uncertainty of $10 \%$, which represents the typical uncertainty for the amplitude in case of the VLBA. Based on our sample of $43 \mathrm{GHz}$ observations we estimated the uncertainty of amplitude by means of gscale statistics in difmap to be slightly higher with an uncertainty of $14 \%$. We derived the standard deviation of gscale values for all intermediate frequencies and antennas assuming a "true" mean value of unity. The uncertainty of amplitude is then given by the median of the final distribution for all observations. However, this analysis includes observations that turned out to be very noisy, as well as observations which are very accurate. In the case of the latter the uncertainty would be largely overestimated. Therefore, we stick to the previously mentioned $10 \%$ uncertainty of amplitude.

To perform a linear regression for deriving speeds, we assigned uncertainties to the data points as follows: the error bar in the width of each model-fit component $m$ was set to $\sigma_{W_{\mathrm{m}}}=W_{\mathrm{m}} /(S / N)_{\mathrm{m}}$, where $(S / N)_{\mathrm{m}}$ is the signal-to-noise ratio $(\mathrm{S} / \mathrm{N})$ of the component peak to the residual noise in the modelfit map, and $W_{\mathrm{m}}$ is the full width half maximum (FWHM) of the Gaussian model-fit component (Fomalont 1999). The error on the position of the Gaussian components depends on the remaining noise in the CLEAN and model-fit maps, size of the components, and beam axis. Therefore, the positional uncertainty was defined as

$\delta \theta=\sqrt{\left(\frac{b_{\mathrm{paj}}}{(S / N)_{\mathrm{c}}}\right)^{2}+\left(\frac{a_{\mathrm{paj}}}{(S / N)_{\mathrm{m}}}\right)^{2}}$,

where $b_{\text {paj }}$ is the beam axis and $a_{\text {paj }}$ is the component axis along the jet position angle (PA), which is equal to $64^{\circ} .(S / N)_{\mathrm{c} / \mathrm{m}}$ is the $\mathrm{S} / \mathrm{N}$ of the map peak to the residual noise in the CLEAN/modelfit map. In the case of very small errors for small component sizes we set a lower boundary to the positional error equal to the beam size divided by 10 , following the approach by Lister et al. (2009).

\section{Results}

There are two periods without observations of about half a year. Over these periods the morphology of the source changes significantly. Hence, we divide the data into three time blocks: Block 1: from 2005 to 2006; Block 2: during 2007; and Block 3: from 2008 to 2009. The individual images, with the Gaussian model fit components plotted on top of the CLEAN maps are shown in Figs. A.1 and A.2. 
A.-K. Baczko et al.: Asymmetric jet production in NGC 1052

Table 1. Image parameters for all analyzed observations at $22 \mathrm{GHz}$ and $43 \mathrm{GHz}$ with natural weighting.

\begin{tabular}{|c|c|c|c|c|c|c|c|c|}
\hline Epoch & VLBA code & $\begin{array}{l}\text { Frequency } \\
(\mathrm{GHz}) \\
(3)\end{array}$ & $\begin{array}{c}\mathrm{rms} \\
\left(\frac{\text { mJy }}{\text { beam }}\right) \\
(4)\end{array}$ & $\begin{array}{c}S_{\text {peak }} \\
\left(\frac{\text { Jy }}{\text { beam }}\right) \\
(5)\end{array}$ & $\begin{array}{l}S_{\text {tot }} \\
(\mathrm{Jy}) \\
(6)\end{array}$ & $\begin{array}{c}b_{\text {maj }} \\
\text { (mas) } \\
(7)\end{array}$ & $\begin{array}{c}b_{\min } \\
(\mathrm{mas}) \\
(8)\end{array}$ & $\begin{array}{l}\mathrm{PA} \\
\left({ }^{\circ}\right) \\
(9)\end{array}$ \\
\hline \multirow[t]{2}{*}{$2005-03-14^{\dagger}$} & BR099A & 43 & 1.21 & 0.33 & 0.80 & 0.46 & 0.20 & 7.37 \\
\hline & & 22 & 8.33 & 0.27 & 0.84 & 0.72 & 0.30 & 4.64 \\
\hline \multirow[t]{2}{*}{$2005-04-22^{\star} \dagger$} & BR009B & 43 & 1.23 & 0.29 & 1.64 & 1.11 & 0.21 & -18.56 \\
\hline & & 22 & 0.70 & 0.53 & 2.09 & 2.32 & 0.36 & -18.58 \\
\hline \multirow[t]{2}{*}{ 2005-06-06 } & BR099C & 43 & 0.99 & 0.41 & 1.17 & 0.69 & 0.20 & -16.85 \\
\hline & & 22 & 0.68 & 0.64 & 2.26 & 0.96 & 0.37 & -10.34 \\
\hline \multirow{2}{*}{ 2005-07-18 } & BR099D & 43 & 0.75 & 0.52 & 1.00 & 0.52 & 0.18 & -11.36 \\
\hline & & 22 & 0.50 & 0.21 & 1.08 & 0.80 & 0.31 & -4.86 \\
\hline \multirow[t]{2}{*}{ 2005-08-22 } & BR099E & 43 & 1.20 & 0.47 & 1.51 & 0.51 & 0.18 & -12.80 \\
\hline & & 22 & 0.85 & 0.58 & 1.99 & 0.89 & 0.32 & -9.62 \\
\hline \multirow[t]{2}{*}{ 2005-10-07 } & BR099F & 43 & 1.05 & 0.48 & 1.30 & 0.49 & 0.19 & -8.47 \\
\hline & & 22 & 0.75 & 0.44 & 1.39 & 0.86 & 0.33 & -6.25 \\
\hline \multirow[t]{2}{*}{ 2005-11-13 } & BR099G & 43 & 0.68 & 0.26 & 0.80 & 0.50 & 0.18 & -9.88 \\
\hline & & 22 & 0.31 & 0.16 & 0.95 & 0.92 & 0.33 & -9.04 \\
\hline \multirow[t]{2}{*}{$2005-12-18^{\star} \dagger$} & BR009H & 43 & 1.02 & 0.40 & 0.91 & 0.64 & 0.20 & -11.64 \\
\hline & & 22 & 0.55 & 0.40 & 0.94 & 1.09 & 0.34 & -10.05 \\
\hline \multirow[t]{2}{*}{ 2006-01-15 } & BR099I & 43 & 0.84 & 0.32 & 0.78 & 0.51 & 0.20 & -8.11 \\
\hline & & 22 & 2.00 & 0.29 & 1.30 & 0.70 & 0.26 & -7.23 \\
\hline \multirow[t]{2}{*}{ 2006-03-06 } & BR119A & 43 & 0.93 & 0.40 & 0.83 & 0.52 & 0.20 & -8.12 \\
\hline & & 22 & 5.58 & 0.36 & 1.28 & 0.65 & 0.26 & -2.84 \\
\hline \multirow[t]{2}{*}{$2006-04-19^{\dagger}$} & BR119B & 43 & 0.74 & 0.41 & 1.04 & 0.48 & 0.18 & -7.15 \\
\hline & & 22 & 1.69 & 0.19 & 1.23 & 0.65 & 0.25 & -4.03 \\
\hline \multirow[t]{2}{*}{$2007-01-14^{\dagger}$} & BR120A & 43 & 0.41 & 0.21 & 0.74 & 0.61 & 0.26 & 10.05 \\
\hline & & 22 & 0.30 & 0.21 & 0.61 & 0.86 & 0.36 & -3.54 \\
\hline \multirow[t]{2}{*}{ 2007-03-04 } & BR120B & 43 & 0.35 & 0.24 & 0.69 & 0.41 & 0.17 & -3.73 \\
\hline & & 22 & 0.27 & 0.23 & 0.67 & 0.89 & 0.36 & -2.42 \\
\hline \multirow[t]{2}{*}{ 2007-05-05 } & BR120C & 43 & 0.42 & 0.25 & 0.49 & 0.40 & 0.16 & -5.15 \\
\hline & & 22 & 0.35 & 0.26 & 0.59 & 0.78 & 0.31 & -5.11 \\
\hline \multirow[t]{2}{*}{ 2007-06-17 } & BR120D & 43 & 0.38 & 0.30 & 0.64 & 0.51 & 0.17 & -8.27 \\
\hline & & 22 & 0.32 & 0.28 & 0.70 & 0.93 & 0.32 & -5.73 \\
\hline 2007-08-11 & BR120E & 43 & 0.44 & 0.26 & 0.56 & 0.53 & 0.18 & -12.30 \\
\hline & & 22 & 0.32 & 0.27 & 0.63 & 0.84 & 0.33 & -4.99 \\
\hline $2007-10-01^{\dagger}$ & BR120F & 43 & 0.40 & 0.29 & 0.63 & 0.88 & 0.49 & 21.76 \\
\hline & & 22 & 0.30 & 0.29 & 0.60 & 1.40 & 0.39 & -16.64 \\
\hline $2008-01-11^{\star} \dagger$ & BR120G & 43 & 0.67 & 0.28 & 0.66 & 0.59 & 0.20 & -11.12 \\
\hline & & 22 & 0.17 & 0.22 & 0.50 & 0.96 & 0.34 & -5.99 \\
\hline 2008-04-04 & BR120H & 43 & 0.37 & 0.21 & 0.73 & 0.54 & 0.19 & -11.98 \\
\hline & & 22 & 0.19 & 0.29 & 0.72 & 1.22 & 0.37 & -15.58 \\
\hline $2008-05-27$ & BR120I & 43 & 0.77 & 0.27 & 0.58 & 0.48 & 0.20 & -4.82 \\
\hline & & 22 & 0.21 & 0.24 & 0.64 & 0.92 & 0.33 & -8.59 \\
\hline 2008-05-04 & BR130A & 43 & 0.56 & 0.30 & 0.70 & 0.42 & 0.16 & -6.07 \\
\hline & & 22 & 0.24 & 0.25 & 0.72 & 0.87 & 0.31 & -8.35 \\
\hline 2008-06-16 & BR130B & 43 & 0.55 & 0.27 & 0.63 & 0.50 & 0.21 & -2.34 \\
\hline & & 22 & 0.24 & 0.24 & 0.65 & 0.86 & 0.33 & -6.65 \\
\hline 2008-07-26 & BR130C & 43 & 0.70 & 0.36 & 0.64 & 0.47 & 0.20 & -4.37 \\
\hline & & 22 & 0.31 & 0.20 & 0.52 & 0.77 & 0.29 & -6.67 \\
\hline 2008-09-06 & BR130D & 43 & 0.77 & 0.31 & 0.69 & 0.59 & 0.25 & -5.82 \\
\hline & & 22 & 0.27 & 0.16 & 0.67 & 0.95 & 0.32 & -10.85 \\
\hline $2008-10-27^{\dagger}$ & BR130E & 43 & 0.47 & 0.11 & 0.78 & 0.44 & 0.18 & -4.77 \\
\hline & & 22 & 0.24 & 0.21 & 0.95 & 0.89 & 0.32 & -8.94 \\
\hline $2008-12-05^{\dagger}$ & BR130F & 43 & 0.50 & 0.22 & 0.72 & 0.76 & 0.32 & 15.87 \\
\hline & & 22 & 0.20 & 0.17 & 0.86 & 0.90 & 0.33 & -7.99 \\
\hline $2009-01-18^{\dagger}$ & BR130G & 43 & 0.50 & 0.26 & 0.69 & 0.99 & 0.37 & 22.77 \\
\hline & & 22 & & & & & & \\
\hline 2009-03-08 & $\mathrm{BR} 130 \mathrm{H}$ & 43 & 0.41 & 0.15 & 0.64 & 0.44 & 0.18 & -3.25 \\
\hline & & 22 & 0.26 & 0.18 & 0.71 & 0.91 & 0.34 & -4.37 \\
\hline 2009-04-16 & BR130I & 43 & 0.32 & 0.16 & 0.65 & 0.43 & 0.16 & -7.43 \\
\hline & & 22 & 0.21 & 0.26 & 0.82 & 0.90 & 0.34 & -6.9 \\
\hline
\end{tabular}

Notes. (1) Date of VLBA observation, ${ }^{(\star)}$ : excluded in $43 \mathrm{GHz}$ analysis, ${ }^{(\dagger)}$ : excluded in combined $43 \mathrm{GHz}$ and $22 \mathrm{GHz}$ analysis; (2) VLBA experiment code; (3) frequency; and (4) rms noise level of image. The RMS was derived in isis by fitting a Gaussian profile to the distribution of pixel values in the images; the rms corresponds to the sigma of the best-fit profile. (5) Peak flux density, (6) total flux density, (7) FWHM major axis of restoring beam, (8) FWHM minor axis of restoring beam, and (9) PA of major axis of restoring beam. 

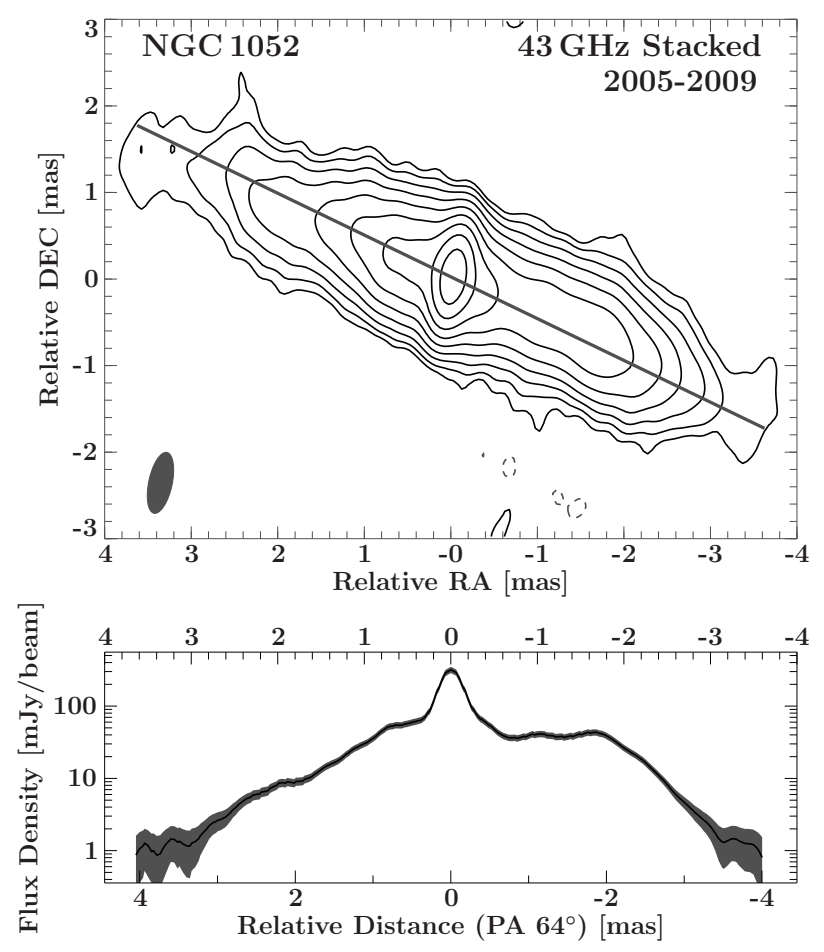

Fig. 1. Stacked image for all observations from 2005 to 2009 (top panel) and flux density along a cut in the jet direction of $64^{\circ}$ (bottom panel). This cut is indicated as a line along the jet axis in the contour map. The common beam for the stacked map is show in the lower left corner of the top panel. For the stacked image, all maps are summed up and divided by the number of images. The contours start at 5 times the noise level and increase logarithmically by factors of 2 .

\subsection{Stacking}

After aligning all images to the bright central peak, we produced a stacked image by adding all analyzed $43 \mathrm{GHz}$ observations pixel by pixel followed by averaging (see Fig. 1) similar to the procedure described in Pushkarev et al. (2017). Both jets are fairly symmetric and have an extent of around 4 mas at this wavelength. A cut along the jet PA at $64^{\circ}$ (from north to east) gives the flux density profile along the jet axes and is illustrated in the lower panel. It forms a plateau from -1 mas to -2 mas along the cut, after which the flux density of the western jet drops off more steeply than the eastern jet.

\subsection{Spectral index maps between $22 \mathrm{GHz}$ and $43 \mathrm{GHz}$}

We made use of simultaneous $22 \mathrm{GHz}$ observations to study the spectral index distribution of the twin-jet system between $43 \mathrm{GHz}$ and $22 \mathrm{GHz}$ (Sect. 3.2.2). Prior to calculating the spectral indices, the images at both frequencies were aligned (Sect. 3.2.1) and convolved with a common beam. Four observations were found to have insufficient image quality for a proper alignment and further five observations did not have a reliable Gaussian model fit at $22 \mathrm{GHz}$ and thus were excluded. After the exclusion of these epochs we were able to obtain 19 epochs, which remain available for a reliable spectral index analysis.

\subsubsection{Alignment}

We used optically thin features in both jets to align the maps at both frequencies (Kadler et al. 2004b; Fromm et al. 2013). The most suitable feature for the alignment is located at around -2 mas in RA distance to the eastern jet core in the $22 \mathrm{GHz}$ map.
Figure 2 gives an example of the alignment applied for the beginning, middle, and end of the observational campaign. These three maps are a good representation of the typical morphology during the individual time blocks and reveal easy to compare Gaussian model fit components. In case of the $22 \mathrm{GHz}$ images we used the Gaussian model fits produced by Böck (2012), which are distributed similarly to the components at $43 \mathrm{GHz}$. For the alignment the beam parameters were not changed (see Table 1 for the individual parameters).

The alignment of the maps sets the central peak in the $43 \mathrm{GHz}$ map around 0.6 mas to the west of the $22 \mathrm{GHz}$ eastern jet peak. For all three examples, the $22 \mathrm{GHz}$ model reveals one Gaussian component at the position of the $43 \mathrm{GHz}$ central component. This is the case for a large percentage of the 19 epochs used in this work, although the $22 \mathrm{GHz}$ central component is substantially fainter and less well defined than the corresponding $43 \mathrm{GHz}$ component.

The four years of observations reveal a rapidly changing twin-jet system. This makes our results difficult to compare with earlier observations. For example, in a multifrequency observation in 2000, Sawada-Satoh et al. (2008) identified the brightest feature at $43 \mathrm{GHz}$ with the eastern jet core at $22 \mathrm{GHz}$. A nearby western component, identified as $\mathrm{C} 4$, resides close to the location where the central engine is suspected. Accounting the difference in flux density to the variability of the jets, it may well be identified with the central feature from our VLBA images.

\subsubsection{Spectral analysis}

After aligning the epochs as discussed in Sect. 3.2.1, spectral index maps are produced based on the hybrid images. Representative spectral images are shown in the right column of Fig. 2 and the common beams for both frequencies used for each map are listed in Table 2. The spectral index distributions reveal significant absorption in the region of the gap at $22 \mathrm{GHz}$, reaching spectral indices of $\alpha=4$ in individual maps. This supports previous results of a free-free absorbing torus covering parts of the western jet (Kameno et al. 2001; Sawada-Satoh et al. 2008) up to $43 \mathrm{GHz}$ (Vermeulen et al. 2003; Kadler et al. 2004b).

Figure 3 shows a spectral index map derived from one stacked map for all 19 epochs at both frequencies. Before stacking, the images were restored with a common beam and images were aligned as discussed in the previous section.

\subsection{Flux density and kinematics}

\subsubsection{Flux density evolution}

The evolution of the flux density over time is tracked by summing up the Gaussian model components of the core as well as components of the western and eastern jets. The evolution of the total flux density and the flux density for each region at $43 \mathrm{GHz}$ is plotted in panels a and b of Fig. 4. NGC 1052 shows significant variability over the four years of our observations. The total flux density rises to a peak of $1.6 \mathrm{Jy}$ in 2005 August, declines below 0.8 Jy during 2007 April, and remains at this level for the rest of the observations.

The flux density evolutions of the core as well as the western and eastern jets follow the trend of the total intensity evolution. In contrast to previous observations in the years 1995-2002, and 2004 (Vermeulen et al. 2003; Kadler et al. 2004b; Baczko et al. 2016a), the western jet is brighter than the eastern jet (see c in Fig. 4) for the whole period 2005-2009. Because of this deviation we assumed the western jet to approach and the eastern jet to recede. 

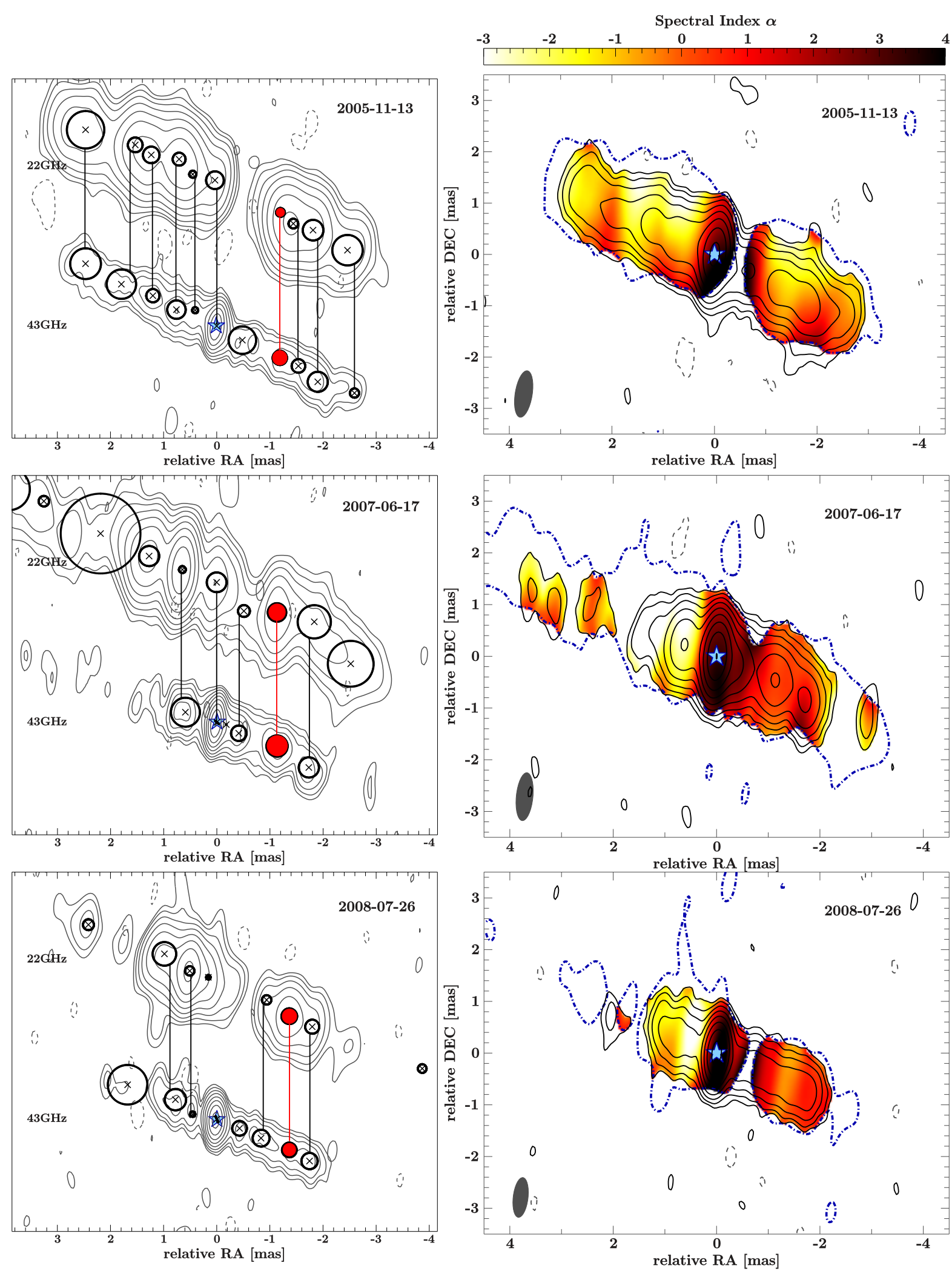

Fig. 2. Left panels: three examples of the alignment of the $22 \mathrm{GHz}$ and $43 \mathrm{GHz}$ maps. We used the optically thin feature $\sim 2$ mas to the west of the $22 \mathrm{GHz}$ jet core (highlighted in red) to derive the relative shift between the two frequencies. The Gaussian model components are plotted on top of the contour clean maps. Vertical lines connect the components identified between both frequencies. The contours start at 3 times the noise level and increase logarithmically by factors of 2 . The beam parameters for the 6 observations are listed in Table 1. Right panels: resulting spectral index maps between $22 \mathrm{GHz}$ (dotted dashed blue contours) and $43 \mathrm{GHz}$ (black contours) after applying the shifts on optically thin features. The restoring beam for each epoch is plotted in the lower left corner of the maps; the parameters are listed in Table 2. The blue star indicates the assumed location of the dynamical center. 
Table 2. Parameters used for the common beam in the spectral index maps shown in Fig. 2.

\begin{tabular}{lccc}
\hline \hline Epoch & $\begin{array}{c}b_{\text {maj }} \\
(\text { mas })\end{array}$ & $\begin{array}{c}b_{\text {min }} \\
(\text { mas })\end{array}$ & $\begin{array}{c}\text { PA } \\
\left({ }^{\circ}\right)\end{array}$ \\
$(1)$ & $(2)$ & $(3)$ & $(4)$ \\
\hline $2005-11-13$ & 0.92 & 0.33 & $-9,04$ \\
$2007-06-17$ & 0.93 & 0.32 & -5.73 \\
$2008-07-26$ & 0.77 & 0.29 & -6.67 \\
\hline
\end{tabular}

Notes. (1) Date of VLBA observation, (2) FWHM major axis of restoring beam, (3) FWHM minor axis of restoring beam, and (4) PA of major axis of restoring beam.

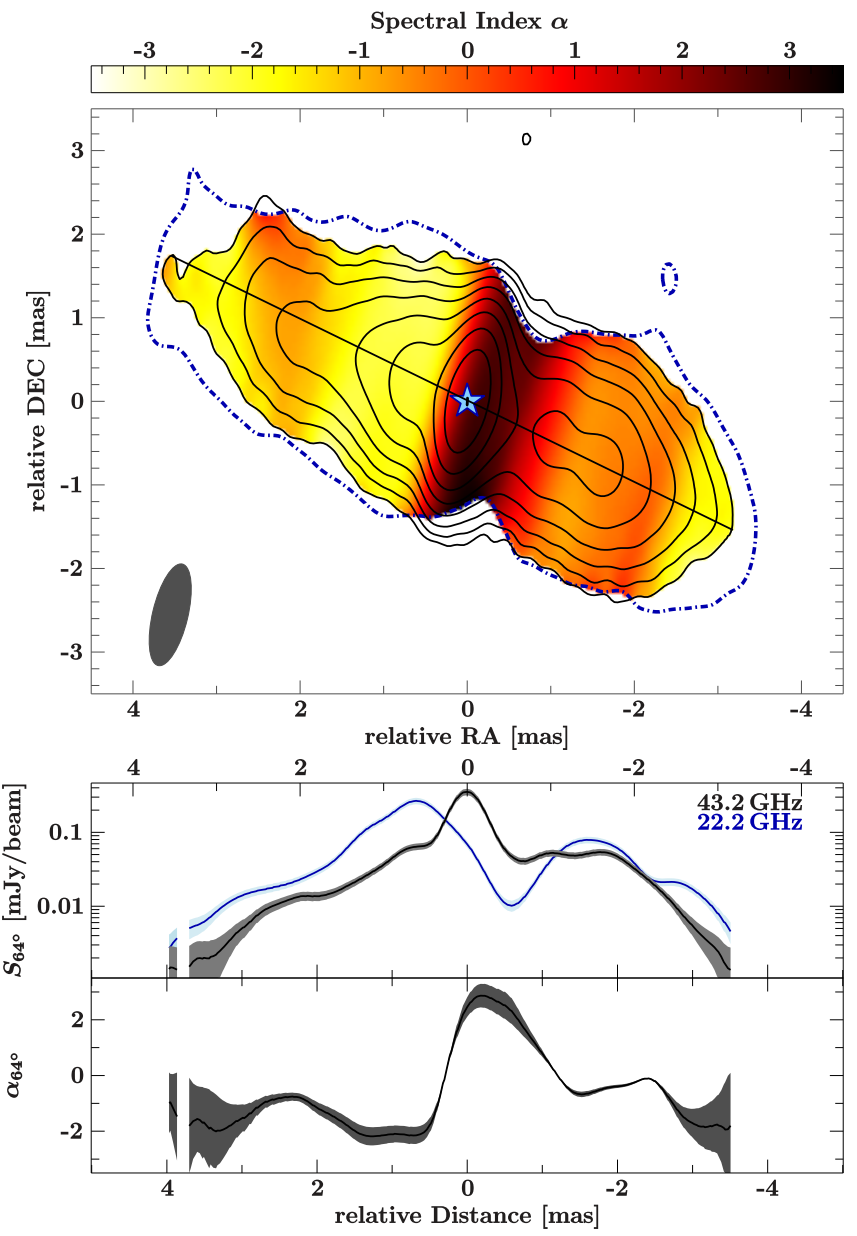

Fig. 3. Stacked spectral index map $\left(S \propto v^{+\alpha}\right)$ combining 19 $22 \mathrm{GHz}$ (blue dash-dotted contour) and $43 \mathrm{GHz}$ (black contours) images between 2005 and 2009. A cut along the direction of the jets is shown in the lower panels, depicting the flux density profile and spectral index along the jet axis (see line in top panel). The blue star indicates the assumed location of the dynamical center. The contours of the $43 \mathrm{GHz}$ map start at 5 times the noise level and increase logarithmically by factors of 2 . For the $22 \mathrm{GHz}$ map only one countour at 5 times the noise level is shown.

We compared our results gained from the analysis of the Gaussian model fits to the initial CLEAN images at $43 \mathrm{GHz}$ and $22 \mathrm{GHz}$ by deriving the flux density evolution based on the distribution of positive CLEAN components in the images at $43 \mathrm{GHz}$ and $22 \mathrm{GHz}$. We included nineteen observations in this comparison, analogous to the spectral analysis in Sect. 3.2. The total flux density and ratio of the brighter to fainter jet is shown in Fig. 5. Similar to Sect. 3.2.2 we first apply the shifts between

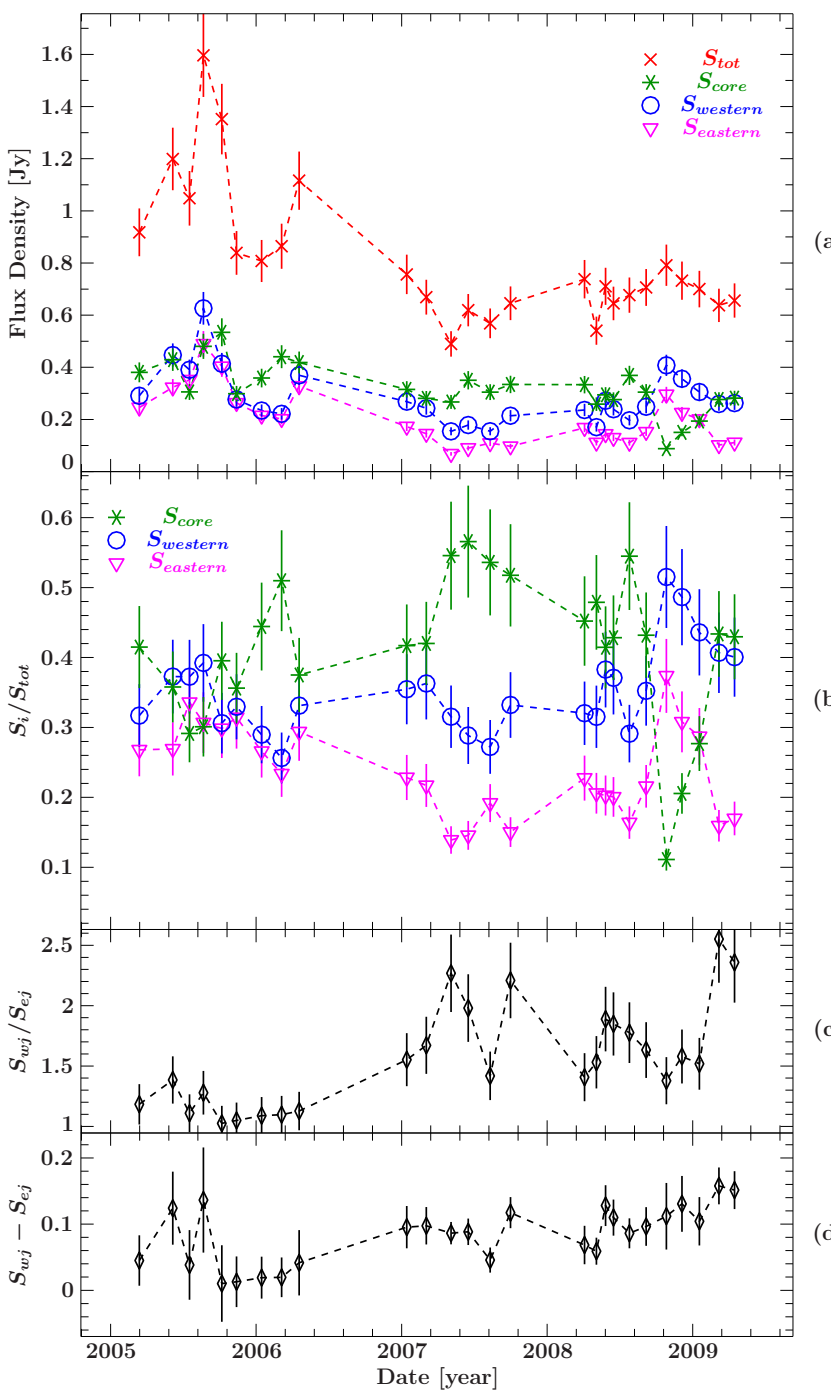

(a)

(b)

Fig. 4. Panel $a$ : light curve at $43 \mathrm{GHz}$ of the total flux density (red), core flux density (green), western jet flux density (blue), and eastern jet flux density (pink). Panel $b$ : fractional intensities (relative to total) of the core component (green), western jet (blue), and eastern jet (pink). Panel $c$ : ratio of the western to eastern jet flux densities. Panel $d$ : absolute difference of the flux density of the western and eastern jet.

the $22 \mathrm{GHz}$ and $43 \mathrm{GHz}$ maps, before excluding CLEAN components up to 0.8 mas distance in east and west to the $43 \mathrm{GHz}$ core at both frequencies to include only the optically thin jet regions. In this way we make sure that we only include the parts of the jets at both frequencies that are, indeed, comparable. All remaining CLEAN components have been summed up for the eastern and western jets.

The comparison of Figs. 4 and 5 shows that both models (Gaussian and CLEAN) result in a consistent evolution of the flux density with time. However, without the need to exclude epochs due to insufficient resolution at $22 \mathrm{GHz}$ Fig. 4 has a more dense time sampling. In contrast to $43 \mathrm{GHz}$ we only find four epochs at $22 \mathrm{GHz}$ in which the western jet is brighter than the eastern jet (2005-06-06, 2005-08-22, 2005-10-07, and 2009-04-16).

\subsubsection{Kinematic analysis}

We used the Gaussian model components in the $2643 \mathrm{GHz}$ maps to examine the dynamics in both jets. We assumed the central peak, observable at all epochs at $43 \mathrm{GHz}$ is the dynamical cen- 


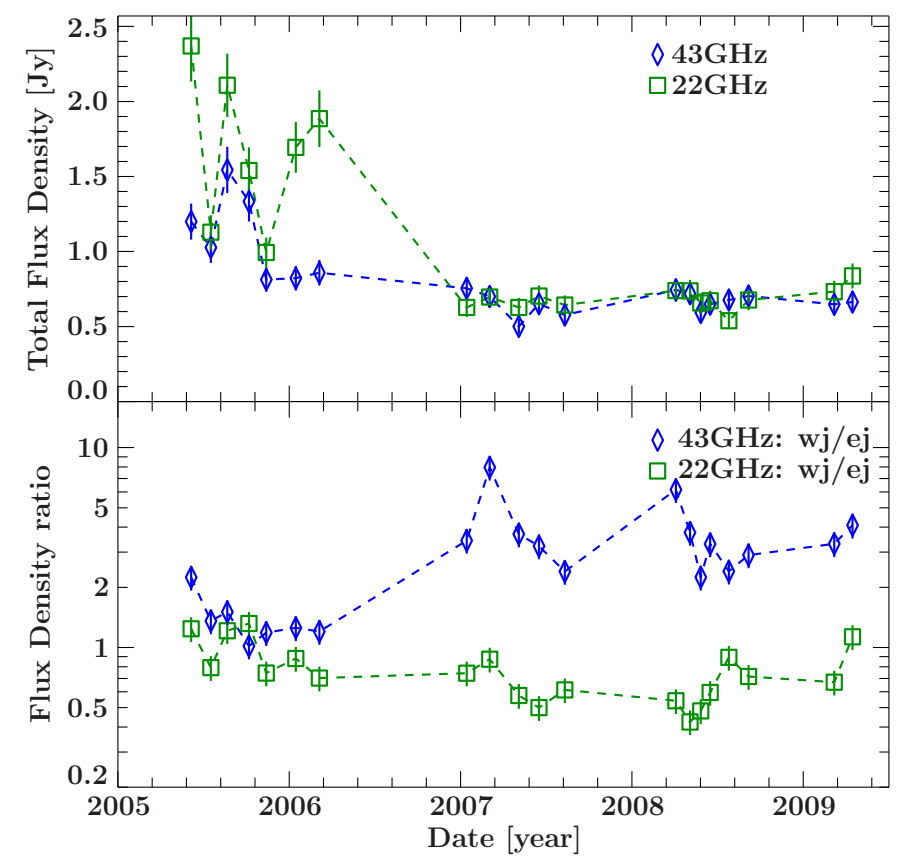

Fig. 5. Top panel: total flux density of 19 clean maps at $22 \mathrm{GHz}$ (green squares) and $43 \mathrm{GHz}$ (blue diamonds). Bottom panel: Flux density ratio of western to eastern jet at $22 \mathrm{GHz}$ (green squares) and $43 \mathrm{GHz}$ (blue diamonds).

ter of the source. This is a natural choice based on the clear morphology at $43 \mathrm{GHz}$ and $86 \mathrm{GHz}$ (cf. Fig. 4 in Baczko et al. 2016 b). In the unlikely case, that the $43 \mathrm{GHz}$ core is offset from the dynamical center, we still would expect that the $43 \mathrm{GHz}$ core does not shift with time. The same holds for the dynamical center and therefore the results obtained in this section are not affected. The Gaussian component fitted to this feature was therefore used to align the maps. The Gaussian model components were cross-identified between adjacent observing epochs. This enabled us to apply linear fits to this feature's core distance as a function of time (see Fig. 6). The jet system is rapidly changing, therefore, we analyzed the three time blocks separately.

We considered only components found in at least four epochs. This results in only one derived velocity for the eastern jet during block 2: ej 10. Together with component wj15 its maximum separation from the center is $<1$ mas and shows a low velocity compared to the other tracked components. It is difficult to decouple their properties from the core region. As a result, both components are not considered in our analysis. Therefore, we do not derive a mean speed for the eastern jet in Block 2 .

The overall mean, maximum, and minimum values for each jet and the mean values for each time block and both jets are listed in Table 3. The uncertainties represent the standard deviation from the mean, assuming an uncertainty on the position of the components as defined in Sect. 2. We derived $\beta_{\mathrm{wj}}=$ $0.343 \pm 0.037$ and $\beta_{\mathrm{ej}}=0.529 \pm 0.038$ for the western and eastern jet, respectively.

\subsection{Brightness temperature and opening angle}

Following the notation of Kadler et al. (2004b), the brightness temperature gradient along the jet axis can be approximated by a power law $T_{\mathrm{b}} \propto r^{s}$ with the power-law index $s=d+n+$ $b\left(1-\alpha_{\mathrm{s}}\right)$, where $\alpha_{\mathrm{s}}$ is the spectral index and assuming that the magnetic field $B$, the electron density $N$, and the jet diameter $D$ are described by power laws as $B \propto r^{b}, N \propto r^{n}$, and $D \propto r^{d}$.
Orthogonal distance regression fits were applied to the brightness temperature and component size of the Gaussian model components with distance from the core. Given the straightness of the jets and the good agreement of jet width with model components, the size of the model components is assumed to represent the width of the jets. In order to avoid confusion from the core region, all components closer than 0.2 mas to the core were excluded. There are a few components with unphysical delta-like sizes leading to a very hight brightness temperature, which exceeds the inverse Compton limit. These had been excluded as well. We assigned uncertainties as discussed in Sect. 2.

As seen in Fig. 7, the brightness temperatures, and jet diameter do not show a simple power-law-like behavior. However, we observe trends in the gradients $s$ and $d$ for the brightness temperature and the opening angle, respectively. When fit with a single power law from 0.2 mas to 4 mas we derive the following index values for the eastern jet: $s_{\mathrm{ej}}=-0.97 \pm 0.15$ and $d_{\mathrm{ej}}=0.03 \pm 0.09$. The western jet shows a break in the $T_{\mathrm{b}}$ distribution and jet diameter. In the outer part, the slopes are steeper than in the inner part. Applying two power laws (red lines in Fig. 7) from 0.2 mas to 0.9 mas and from 0.9 mas to 4.0 mas results in the parameters listed in Table 5, suggesting a conical expansion in the outer part for the western jet.

There are several components with small sizes that do not seem to be a good representation of the width of the jets, but rather maybe due to local substructures within the jets (see Figs. A.1 and A.2). We excluded all model-fit components with sizes below 0.15 mas (gray diamonds in Fig. 7) and fit power laws to both regions $(0.2-0.9$ mas and $0.9-4$ mas) for both jets (blue lines in Fig. 7). The power-law indices are listed in the bottom part of Table 5 .

\section{Discussion}

\subsection{Torus}

The stacked spectral index map (see Sect. 3.2.2 and Fig. 3) reveals an absorbing structure near the base of the two jets. The absorber covers a region of about 0.4 mas $(0.04 \mathrm{pc})$ to the east and 0.8 mas $(0.08 \mathrm{pc})$ to the west from the $43 \mathrm{GHz}$ map peak. The spectral index $\alpha$ in this region exceeds the theoretical upper limit for synchrotron self-absorption of 2.5 . The $43 \mathrm{GHz}$ core is very close to the peak of the absorbing structure and therefore we do not expect a significant offset from the dynamic center. Furthermore, both jets show a relatively steep spectrum and have a mean spectral index around -1 , which further decreases to a minimum of $\sim-2$. This is in agreement with the values reported by Kadler et al. (2004b) of around -1 in both jets, which suggests that the outer jets are optically thin. It is possible to produce images at both frequencies that show no gap in the emission using the stacked map. This allows us to give a more continuous measurement of the geometry of the absorbing medium than possible in previous works (see, e.g., Kellermann et al. 1999; Kameno et al. 2001; Vermeulen et al. 2003; Kadler et al. 2004b).

Kameno et al. (2001) derived the distribution of the FFA opacity due to the torus and found a peak opacity at $1 \mathrm{GHz}$ of $\tau_{\mathrm{f}, 1 \mathrm{GHz}}=300$ in the nucleus. The FFA opacity coefficients derived by Sawada-Satoh et al. (2008) are consistent with this, but show a higher mean optical depth in the center of $\tau_{\mathrm{f}, 1 \mathrm{GHz}}=$ 1000. Given a frequency dependence of the optical depth as $\tau_{\mathrm{f}} \propto v^{-2.1}$ the free-free opacity at $43 \mathrm{GHz}$ can be estimated to $\tau_{\mathrm{f}, 43 \mathrm{GHz}}=0.11$ for assuming $\tau_{\mathrm{f}, 1 \mathrm{GHz}}=300$ and to $\tau_{\mathrm{f}, 43 \mathrm{GHz}}=$ 


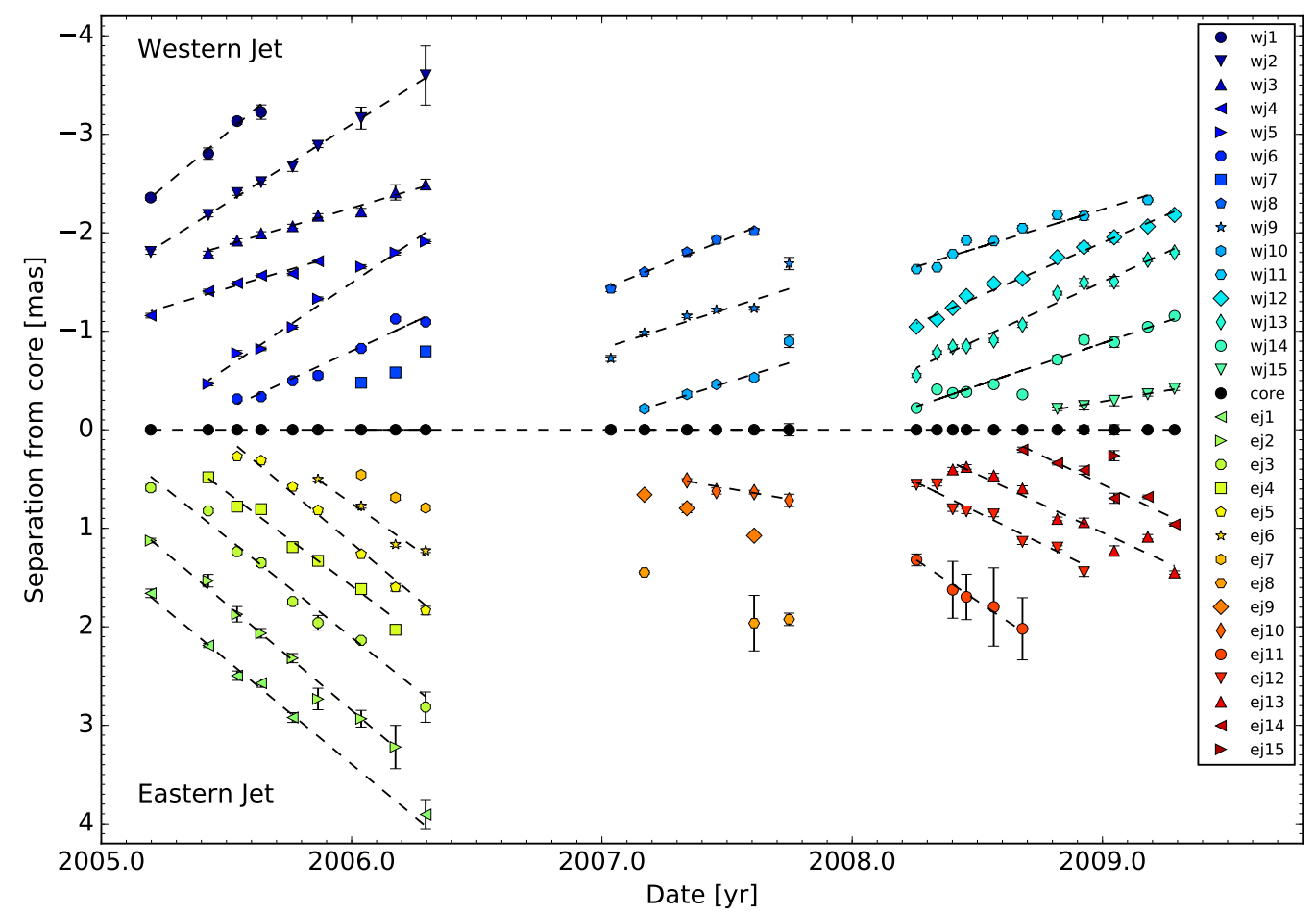

Fig. 6. Core separation of individual Gaussian model-fit components over time. The fit to a linear function for each component is indicated by a dashed line (corresponding slopes are listed in Table 4). The eastern jet is plotted in the lower half of the plot, the western jet in the top half of the plot.

Table 3. Averaged speeds for the western jet (top) and eastern jet (bottom) for each observational time block and mean, minimum, and maximum for all observations for each jet.

\begin{tabular}{lcc}
\hline \hline & \multicolumn{2}{c}{ Western jet } \\
& $v_{\text {mean }}\left(\mathrm{mas} \mathrm{yr}^{-1}\right)$ & $\beta_{\text {mean }}(\mathrm{c})$ \\
\hline Block 1 & $1.361 \pm 0.230$ & $0.412 \pm 0.069$ \\
Block 2 & $0.882 \pm 0.074$ & $0.267 \pm 0.023$ \\
Block 3 & $0.981 \pm 0.093$ & $0.297 \pm 0.028$ \\
Mean & $1.134 \pm 0.122$ & $0.343 \pm 0.037$ \\
Min & $0.753 \pm 0.048$ & $0.228 \pm 0.016$ \\
Max & $2.152 \pm 0.138$ & $0.651 \pm 0.042$ \\
\multicolumn{3}{c}{ Eastern jet } \\
\hline Block 1 & $2.025 \pm 0.060$ & $0.613 \pm 0.018$ \\
Block 2 & \multicolumn{2}{c}{-} \\
Block 3 & $1.336 \pm 0.125$ & $0.404 \pm 0.038$ \\
Mean & $1.749 \pm 0.126$ & $0.529 \pm 0.038$ \\
Min & $1.181 \pm 0.098$ & $0.357 \pm 0.030$ \\
Max & $2.156 \pm 0.055$ & $0.652 \pm 0.017$ \\
\hline
\end{tabular}

0.37 for assuming $\tau_{\mathrm{f}, 1 \mathrm{GHz}}=1000$. The observed flux density at $43 \mathrm{GHz}\left(S_{\mathrm{abs}}(43 \mathrm{GHz})\right)$ is typically around $300 \mathrm{mJy}$ for the core region. It is connected to the intrinsic flux density $\left(S_{\text {int }}(v)\right)$ and $\tau_{\mathrm{f}}$ by $S_{\text {abs }}(v)=S_{\text {int }}(v) \mathrm{e}^{-\tau_{\mathrm{f}}}$. For $S_{\text {abs }}(43 \mathrm{GHz})=300 \mathrm{mJy}$ we get $S_{\text {int }}(43 \mathrm{GHz})=335 \mathrm{mJy}$ and $S_{\text {int }}(43 \mathrm{GHz})=434 \mathrm{mJy}$. Based on these assumptions we cannot assume this region to be optically thin. However, there are very few epochs in which jet components are close enough to the central core to be affected by absorption (for example BR130F or BR130E). These epochs reveal strongly correlated Gaussian model parameters in the innermost jet regions including the core. Therefore, these observations are already not optimal to analyse the innermost region. For all other observations the central component is still the brightest when accounting for absorption and is therefore still the most likely position of the core.

The location of the cut in the power laws in the western jet, as seen in Fig. 7, is consistent with the outer edge of the absorbing torus. If we still assume some absorption at $43 \mathrm{GHz}$, this would result in a reduction of the flux density of the western jet in this region, which would satisfactorily explain the brightness temperature distribution (cf. Kadler et al. 2004a). If the drop in the brightness temperature distribution is only due to the absorption, interpolating the outer gradient inward should give us the real brightness temperatures close to the central region. This results in higher values for the western jet. In addition, the asymmetry observed from 2007 on is even more pronounced.

In comparison, Kadler et al. (2004b) derived power-law index values $s$ between $-3.8 \pm 0.3$ and $-4.1 \pm 0.8$ for the eastern jet at frequencies of $5,8.4,22$, and $43 \mathrm{GHz}$. The brightness temperature distribution of the western jet could not be approximated by a simple power law. The fits were consistent for all frequencies but were performed only for components farther away than 2.5 mas from the center. Kadler et al. (2004b) analyzed only one epoch per frequency, whereas our analysis includes 26 epochs at $43 \mathrm{GHz}$, resulting in a more robust statistics. Adding the derived values by Kadler et al. (2004b) to our analysis, the absolute value of the $T_{\mathrm{b}}$ power-law index increases with increasing distance from the core.

In the numerical simulations of Fromm et al. (2018) initially symmetric jets are assumed. However, asymmetries between the jet and counterjet appear because of the delicate interplay between absorption and orientation of the jet-torus system and the properties of the observing array.

In the case of overpressured jets embedded in a decreasing pressure ambient medium several recollimation shocks can be formed. The observed signature of a recollimation shock can be described by a stationary local flux density maximum, in contrast to traveling shocks where the local increase in flux 
Table 4. Calculated velocities $(v)$ and ejection times (ejt) for the individual components including including errors.

\begin{tabular}{lccc}
\hline \hline ID & $v\left(\right.$ mas yr $\left.^{-1}\right)$ & $\beta_{\text {app }}(\mathrm{c})$ & ejt $(\mathrm{yr})$ \\
\hline ej1 & $2.116 \pm 0.105$ & $0.640 \pm 0.032$ & $2004.945 \pm 0.043$ \\
ej2 & $2.156 \pm 0.055$ & $0.652 \pm 0.017$ & $2005.168 \pm 0.016$ \\
ej3 & $2.034 \pm 0.140$ & $0.615 \pm 0.042$ & $2005.513 \pm 0.040$ \\
ej4 & $1.906 \pm 0.125$ & $0.577 \pm 0.038$ & $2005.543 \pm 0.029$ \\
ej5 & $2.145 \pm 0.141$ & $0.649 \pm 0.042$ & $2005.841 \pm 0.022$ \\
ej6 & $1.790 \pm 0.253$ & $0.542 \pm 0.076$ & $2005.798 \pm 0.056$ \\
ej7 & - & - & - \\
ej8 & - & - & - \\
ej9 & - & - & - \\
ej10 & $0.451 \pm 0.101$ & $0.137 \pm 0.031$ & $2006.389 \pm 0.263$ \\
ej11 & $1.710 \pm 0.095$ & $0.517 \pm 0.029$ & $2007.695 \pm 0.043$ \\
ej12 & $1.245 \pm 0.128$ & $0.377 \pm 0.039$ & $2008.162 \pm 0.055$ \\
ej13 & $1.181 \pm 0.098$ & $0.357 \pm 0.030$ & $2008.560 \pm 0.050$ \\
ej14 & $1.208 \pm 0.131$ & $0.365 \pm 0.040$ & $2008.844 \pm 0.049$ \\
ej15 & - & - & - \\
wj1 & $-2.152 \pm 0.138$ & $0.651 \pm 0.042$ & $2004.322 \pm 0.072$ \\
wj2 & $-1.600 \pm 0.032$ & $0.484 \pm 0.010$ & $2004.611 \pm 0.024$ \\
wj3 & $-0.753 \pm 0.048$ & $0.228 \pm 0.015$ & $2003.446 \pm 0.157$ \\
wj4 & $-0.760 \pm 0.077$ & $0.230 \pm 0.023$ & $2003.946 \pm 0.167$ \\
wj5 & $-1.727 \pm 0.096$ & $0.523 \pm 0.029$ & $2005.571 \pm 0.032$ \\
wj6 & $-1.177 \pm 0.099$ & $0.356 \pm 0.030$ & $2005.698 \pm 0.042$ \\
wj7 & - & - & - \\
wj8 & $-1.031 \pm 0.074$ & $0.312 \pm 0.022$ & $2005.906 \pm 0.104$ \\
wj9 & $-0.814 \pm 0.190$ & $0.246 \pm 0.057$ & $2006.344 \pm 0.260$ \\
wj10 & $-0.802 \pm 0.130$ & $0.243 \pm 0.039$ & $2007.190 \pm 0.065$ \\
wj11 & $-0.784 \pm 0.074$ & $0.237 \pm 0.022$ & $2006.607 \pm 0.204$ \\
wj12 & $-1.104 \pm 0.042$ & $0.334 \pm 0.013$ & $2007.794 \pm 0.042$ \\
wj13 & $-1.173 \pm 0.056$ & $0.355 \pm 0.017$ & $2008.235 \pm 0.037$ \\
wj14 & $-0.862 \pm 0.069$ & $0.261 \pm 0.021$ & $2008.496 \pm 0.052$ \\
wj15 & $-0.435 \pm 0.017$ & $0.132 \pm 0.005$ & $2008.573 \pm 0.023$ \\
\hline & & &
\end{tabular}

density is advected with the plasma. The local increase in pressure and density at the recollimation shocks decreases with distance from the jet nozzle and thus the observed emission signatures of standing recollimation shocks diminish with distance down the jet. Therefore, the strongest recollimation shocks in each jet, which would appear as stationary flux density maxima, should be located to the jet nozzle.

However, depending on the torus properties, the observing frequency and orientation of the jet-torus system these features can be hidden behind the obscuring torus. The obscuring torus becomes optically thinner at higher frequencies offering us a glimpse into the innermost regions of the central engine. As a consequence the local flux density maxima associated with the recollimation shocks may become visible (see Fig. 14 in Fromm et al. 2018). If we assume NGC 1052 has a viewing angle $\vartheta \neq 90$, we expect the larger absorption along the line of sight to result in less flux in one of the jets (i.e., in the counterjet). Also large spectral index values $(\sim 3)$ can be obtained between the jet and counterjet owing to the contribution of the FFA in the torus (see Fig. 16 in Fromm et al. 2018). To test whether the jets are initially symmetric or asymmetric, detailed simulations and modelling tailored to NGC 1052 have to be performed.

\subsection{Jet properties}

\subsubsection{Morphology}

Comparing the first epoch at $43 \mathrm{GHz}$ in 2005 and the $86 \mathrm{GHz}$ map from October 2004 (Baczko et al. 2016a), both maps reveal one central bright feature and two fainter, symmetrically evolving jets. We attempted component identification between both frequency maps. However, there are several difficulties that prevent us from obtaining a robust result: (1) There is a gap of five months between the $86 \mathrm{GHz}$ observation and the first $43 \mathrm{GHz}$ observation. Based on our study, the morphology of the source might change significantly during this time. (2) By extrapolating the western jet component locations to the time of the $86 \mathrm{GHz}$ observation, we find there are several paths that cross. Therefore, it is not clear where the components were located in October 2004. (3) Extrapolating the components in epoch BR099A backward only gives information about the inner 1 mas of the eastern jet, which is not sufficient to compare with the observation at $86 \mathrm{GHz}$. (4) The quality of the $86 \mathrm{GHz}$ data is not good enough to make a reliable association. For this, a model fit degrading the weights of the long-baseline data (tapering) should be considered, since we do not have sufficient $\mathrm{S} / \mathrm{N}$ in the full-resolution image at $3.5 \mathrm{~mm}$.

As seen in Figs. A.1 and A.2, there are remarkable differences between the first observational block and the following two. Therefore, stacked maps for each block have been produced (see Fig. 10). In Block 1 NGC 1052 shows a symmetric morphology, whereas in Block 2 the jets become asymmetric and the source is more compact. The extent of the western jet is about 1 mas smaller than in Block 1 . The eastern jet becomes less prominent in blocks 2 and 3 (see Fig. 10). It shows only diffuse emission beyond 2 mas.

The fits to the jet diameter indicate strict deviations from a conical expansion in the inner 1 mas (corresponding to $0.1 \mathrm{pc}$ ) of both jets. At the point of the change in the power-law index, there is a break in the distribution, resulting in smaller major axes at around 1 mas, indicating a possible re-collimation of the jets.

\subsubsection{Speed}

There is a clear trend toward higher velocities in the eastern jet in comparison to the western jet (see Table 4). For time Block 1 the basic kinematic model was previously presented in Baczko et al. (2016b). The western jet in Block 1 shows a wide spread of velocities. In contrast to that the component velocities in the eastern jet are very similar and have deviations of less than $0.1 \mathrm{c}$ from the mean value. For Block 2 and Block 3 the western jet is more stable, revealing comparable speeds for all tracked components. However, the overall evolution in both jets show that, besides the clear trend toward higher velocities in the eastern jet, there are significant deviations from the mean speed.

We compare the measured apparent velocities at $15 \mathrm{GHz}$ from Lister et al. (2013) with the values at $43 \mathrm{GHz}$ in Fig. 8. The components at $43 \mathrm{GHz}$ have significantly different values than those at $15 \mathrm{GHz}$ with a tendency of higher velocities at higher frequency. As discussed in the previous sections, a circumnuclear torus covers large parts of the western jet, thus FFA results in an obscuration of the inner part at lower frequencies (Kameno et al. 2001; Vermeulen et al. 2003; Kadler et al. 2004b). Its impact at $43 \mathrm{GHz}$ is already small enough so that we are able to peer through this absorbing structure. If the jet has transversal velocity structure, it is likely, that we are observing a faster moving layer of the jets at $43 \mathrm{GHz}$ as compared to lower frequencies. The slower moving layer of the jet, as observable at $15 \mathrm{GHz}$, may very well be invisible at $43 \mathrm{GHz}$ owing to less energetic particles that are not emitting at $43 \mathrm{GHz}$.

\subsubsection{Viewing angle}

An estimate on the angle of the jets to the line of sight $\theta_{\mathrm{LOS}}$ can be derived from their flux-density ratio. If we assume that 

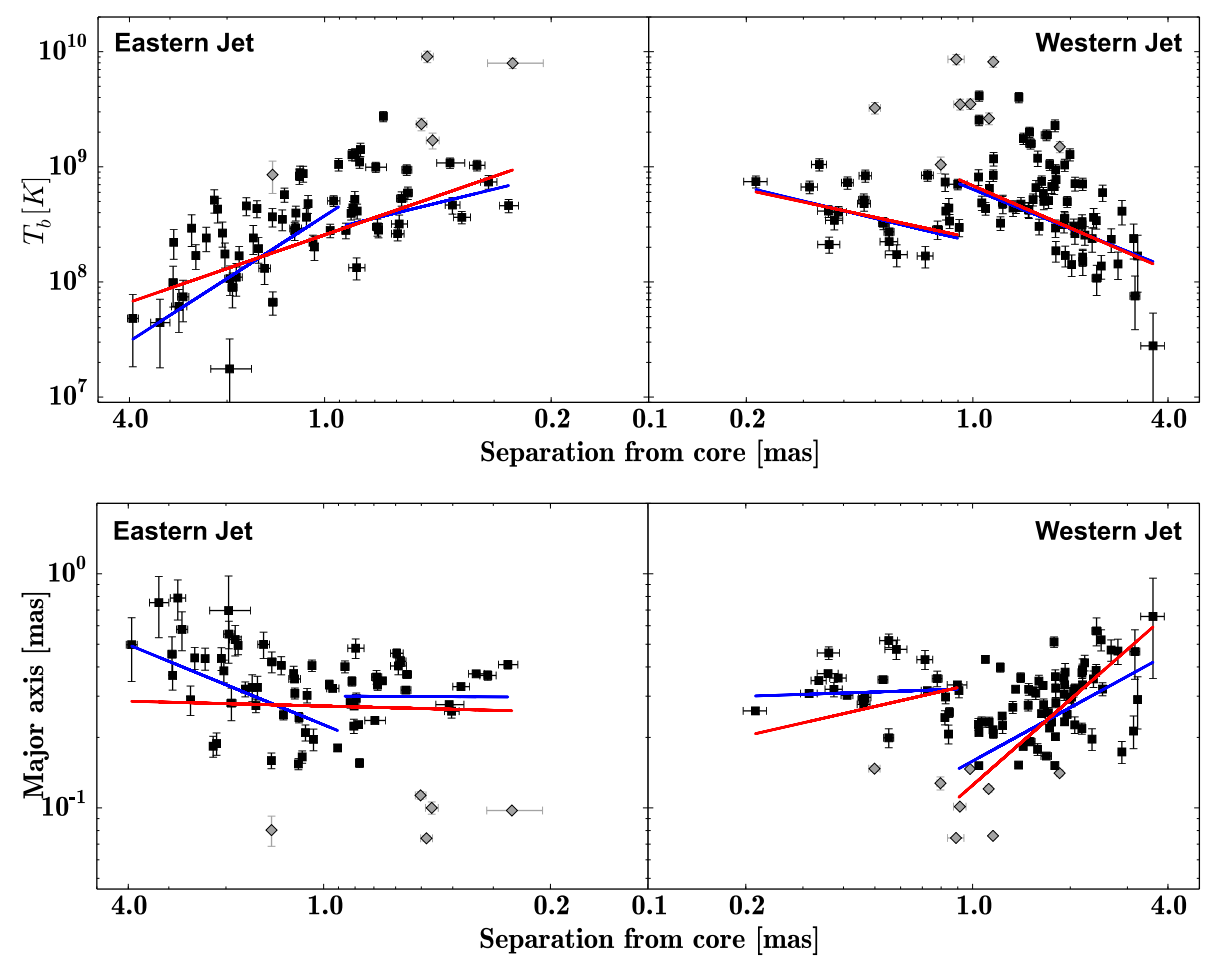

Fig. 7. Brightness temperature (top panel) and component major axis (bottom panel) as functions of the core distance for both jets. Power laws are fitted $\left(T_{\mathrm{b}} \propto r^{s} ; D \propto r^{d}\right)$ and the indices are printed in Table 5. Two cases are distinguished: including all data points (red lines) and excluding the gray diamonds, corresponding to circular Gaussian fits whose major axes are smaller than 0.15 mas in size (blue lines). These data points do not seem to be a good representation of the width of the jets, but rather maybe owing to substructures within the jets.
Table 5. Power-law indices for brightness temperature $\left(T_{\mathrm{b}} \propto r^{s}\right)$ and jet diameter $\left(D \propto r^{d}\right)$ of both jets.

\begin{tabular}{lcccc}
\hline \hline Region (mas) & $s_{\mathrm{wj}}$ & $s_{\mathrm{ej}}$ & $d_{\mathrm{wj}}$ & $d_{\mathrm{ej}}$ \\
\hline \multicolumn{5}{c}{ Fit to all data points } \\
\hline $0.2-4.0$ & - & $-0.97 \pm 0.15$ & - & $0.03 \pm 0.09$ \\
$0.2-0.9$ & $-0.60 \pm 0.40$ & - & $0.32 \pm 0.19$ & - \\
$0.9-4.0$ & $-1.22 \pm 0.29$ & - & $1.22 \pm 0.16$ & - \\
\hline \multicolumn{5}{c}{ Fit to robust data points } \\
\hline $0.2-0.9$ & $-0.68 \pm 0.34$ & $-0.68 \pm 0.37$ & $0.05 \pm 0.07$ & $0.01 \pm 0.10$ \\
$0.9-4.0$ & $-1.13 \pm 0.27$ & $-1.81 \pm 0.39$ & $0.76 \pm 0.14$ & $0.57 \pm 0.20$ \\
\hline
\end{tabular}

Notes. Orthogonal distance regression fits had been applied to the different regions as shown in Fig. 7.

both jets are intrinsically symmetric and evolve with an angle of $180 \mathrm{deg}$ between the two jet axes, i.e.,

$R=\frac{S_{\text {approaching }}}{S_{\text {receding }}}=\left(\frac{1+\beta \cos \theta_{\mathrm{LOS}}}{1-\beta \cos \theta_{\mathrm{LOS}}}\right)^{2-\alpha}$

In this case, $\beta$ is the intrinsic jet speed and $\alpha$ the spectral index. According to Sect. 4.1 we adopt a value of $\alpha=-1$, consistent with our $22-43 \mathrm{GHz}$ observations.

In addition, the angle of both jets to the line of sight can be derived with knowledge of their intrinsic $(\beta)$ and apparent $\left(\beta_{\text {app }}\right)$ speeds for approaching and receding jets as follows:

$\beta_{\text {app }, \text { approaching } / \text { receding }}=\frac{\beta \sin \theta_{\mathrm{LOS}}}{1 \mp \beta \cos \theta_{\mathrm{LOS}}}$.

Both equations can be combined to derive the allowed parameter space for the intrinsic velocity $\beta$ and the angle of the jets to the line of sight $\theta_{\mathrm{LOS}}$ by using the derived values for the velocity and flux density ratios.

As discussed in the previous sections, there are differences in velocity between both jets and the various time blocks. In addition we find the western jet to be consistently brighter

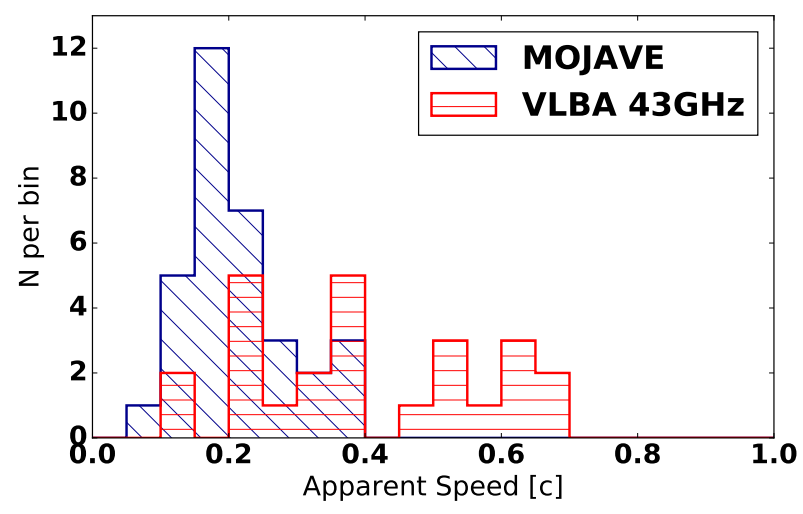

Fig. 8. Histogram of the apparent velocity of the jet components for this work at $43 \mathrm{GHz}$ (red), and Lister et al. (2013; blue, MOJAVE) at $15 \mathrm{GHz}$. The bin size is $0.05 \mathrm{c}$.

than the eastern jet at $43 \mathrm{GHz}$. This result is contradictory to earlier studies, which showed the eastern jet to be brighter (Kellermann et al. 1999; Vermeulen et al. 2003; Kadler et al. 2004a). Our analysis reveals a change in the jet-to-counter-jet ratio over the span of four years from around $R=1.1$ up to $R=2.5$ (see Fig. 4c). For the first observational block, the ratio remains at a similar level of around $R=1.1$; in this period the morphology of the jets appears to be symmetric. In the second observational block (2007) the jets become increasingly asymmetric with a mean ratio of about $R=1.9$.

Based on our results there is evidence that the jets are not symmetric. However, Eqs. (2) and (3) can be used to test this assumption. If symmetry holds, we should be able to obtain a common region in the parameter space for $\theta_{\mathrm{LOS}}$ and $\beta$ that hold for all observations. The eastern jet in observational Block 2 is very short and reveals only very few tracked components, therefore we compare blocks 1 and 3. In Eq. (3) we used the maximum speeds found during the appropriate blocks. Based on the herein discussed $43 \mathrm{GHz}$ observations and the morphology 

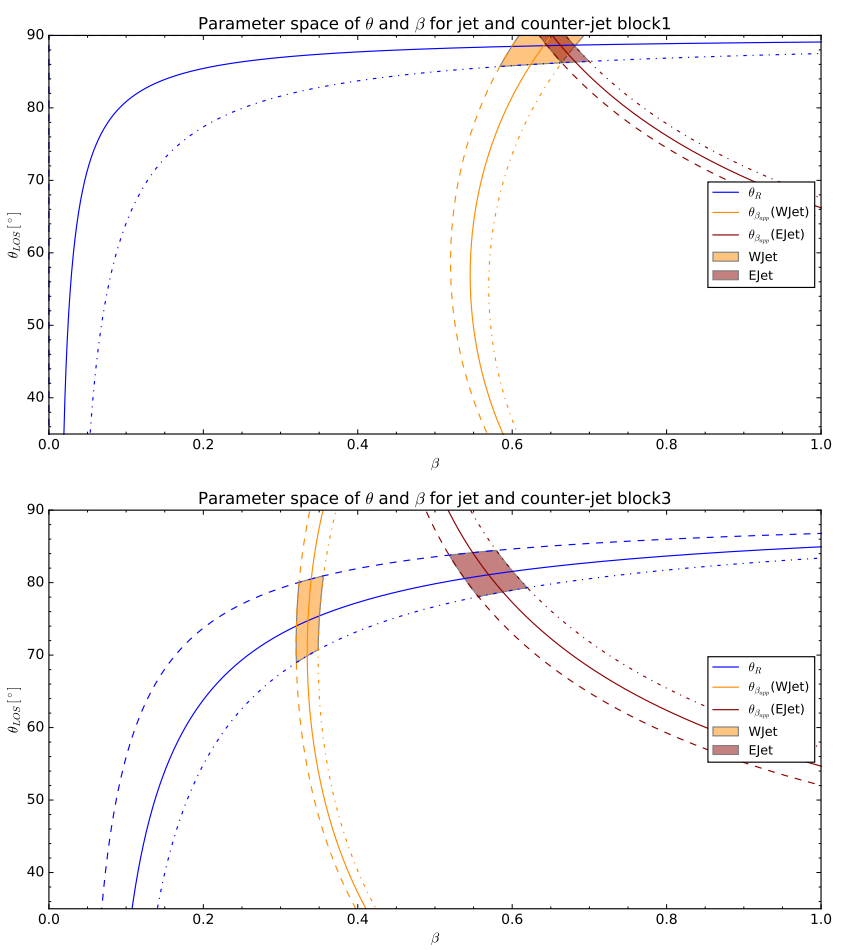

Fig. 9. Angle of the jets to the line of sight $\theta_{\mathrm{LOS}}$ as a function of the intrinsic jet velocity $\beta$, constrained by the western-to-eastern jet ratio $R$ (blue) and the apparent maximum velocities for eastern jet (red) and western jet (orange) for observational blocks 1 and 3 . The allowed parameter space for $\theta_{\mathrm{LOS}}$ and $\beta$ for eastern and western jet is highlighted by the red/orange-shaded region, respectively. The dashed and dash-dotted lines indicate the uncertainty range. We do not find a common parameter space for both jets and both blocks. The assumption of intrinsic asymmetry is therefore contradictory to our results.

outside the central region, we assumed the western jet to be the approaching jet and the eastern jet to be the receding jet. As shown in Fig. 9 we find a common region in the parameter space for $\theta_{\mathrm{LOS}}$ and $\beta$ in Block 1. In Block 3 the velocities depart from each other and cannot be compatible, so it is not possible to combine eastern and western jet assuming intrinsic symmetry.

The assumption that both jets are intrinsically identical in terms of velocity, viewing angle, and an overall symmetric evolution was implicit in our study of the parameter space. However, there is no combination of $\beta$ and $\theta_{\mathrm{LOS}}$ that is consistent for both blocks and jets. This contradicts the assumption of symmetry and therefore at least one equality constraint must be lifted.

\subsection{Source geometry}

The asymmetries we observe may originate from asymmetries either in the physical nature of the jets themselves or the environment into which they propagate. If we assume the drop in $T_{\mathrm{b}}$ in the inner part of the western jet is due to the absorbing structure, the interpolation of the outer gradient results in higher brightness temperatures close to the central engine compared to the eastern jet. This is most likely explained by a higher brightness of the western jet, thus requiring a larger jet internal energy and/or magnetic flux. For example, this can be achieved by a more energetic ejection producing larger particle internal energy and/or magnetic flux. This would favor an internal asymmetry in the jets. On the other hand, even if we assume that the same energy flux is injected at the nozzle of both jets, there can also be disturbances further downstream, resulting in different energy conversion and leading to a possible higher emissivity of the western jet. Given the large scales on which the jets are interacting with the interstellar medium it is most likely that this environment is not identical for both jets. In particular, stronger dissipation or increased mass loading by, for example, stellar winds, can lead to a brighter and slower flow (see, e.g., Bowman et al. 1996; Perucho et al. 2014).

Combining the results from the kinematic analysis at $43 \mathrm{GHz}$ with previous studies at lower frequencies (see, e.g., Vermeulen et al. 2003) further constrains the jet properties and formation is possible. The particles emitting at $43 \mathrm{GHz}$ are assumed to be accelerated from regions of the accretion disk, which are closer to the SMBH. Finally, we expect to observe an inner layer of the jet. This would lead to a faster moving inner layer and a slower outer layer, which is not emitting at $43 \mathrm{GHz}$.

The physical conditions are getting more extreme when coming closer to the central engine. If the direct surrounding of the central SMBH has a larger impact at $43 \mathrm{GHz}$, this would explain the different behavior of the source at this frequency in comparison to lower frequencies. The energy conversion processes are more violent and disturbances can happen on shorter timescales.

\section{Precession or asymmetric jet production?}

As discussed in Sect. 4.2.3 our results do not fit into a simple picture in which we assume the same intrinsic conditions in both jets. There are two main possibilities capable of explaining this. First, jets are not intrinsically identical. Second, if $\beta$ does not change, the flux density ratio only depends on $\theta_{\text {LOS }}$. This would result in a change of $\theta_{\text {LOS }}$ over the four years of observation, implying a precessing jet.

The galactic X-ray binary SS 433 is an example of an precessing jet. The jet precession occurs within a cone of halfopening angle of $19.85^{\circ}$ with respect to the angle to our line of sight of $79^{\circ}$ with a period of 162 days (Margon \& Anderson 1989). As larger scale counterparts to SS 433, radio galaxies have been observed to reveal helical motions as well. In particular, a considerable amount of quasars have S-shaped jets (for example 3C273 Perley \& Meisenheimer 2017 or 3C345 Matveyenko \& Sivakon' 2013). There are several mechanisms leading to a precessing jet. One often discussed scenario is the Bardeen-Petterson (Bardeen \& Petterson 1975) effect, which is due to a misalignment of the angular momentum of the accretion disk and rotating central black hole. Based on this approach jet precession has been successfully described in several blazars (see, e.g., Liu \& Melia 2002; Caproni et al. 2004). Typically very long precession periods are observed.

There is evidence for a recent merger event in NGC 1052 about 1-2 Gyr ago (van Gorkom et al. 1986; Pierce et al. 2005). Therefore, another likely explanation is a binary black hole, where the companion exerts a torque and therefore results in a precession of the accretion disk, resulting in shorter periods of precession.

There are many studies concerning jet precession (see for example Mohan et al. 2016; Machalski et al. 2016; Rozgonyi \& Frey 2016; Qian et al. 2017 and references therein). Examples are $1308+226$ with an orbital period of $\simeq 8.5 \mathrm{yr}$ in case of a binary black hole model (Britzen et al. 2017), 2MASXJ12032061+1319316 with a precession period of $0.95 \times 10^{5} \mathrm{yr}$ (Rubinur et al. 2017), and M 81 with a period of 7.27 yr (Martí-Vidal et al. 2011).

If the jets are initially asymmetric the question arises as to which mechanism is able to produce this behavior. One possible physical scenario is connected to the magnetic field structure in 

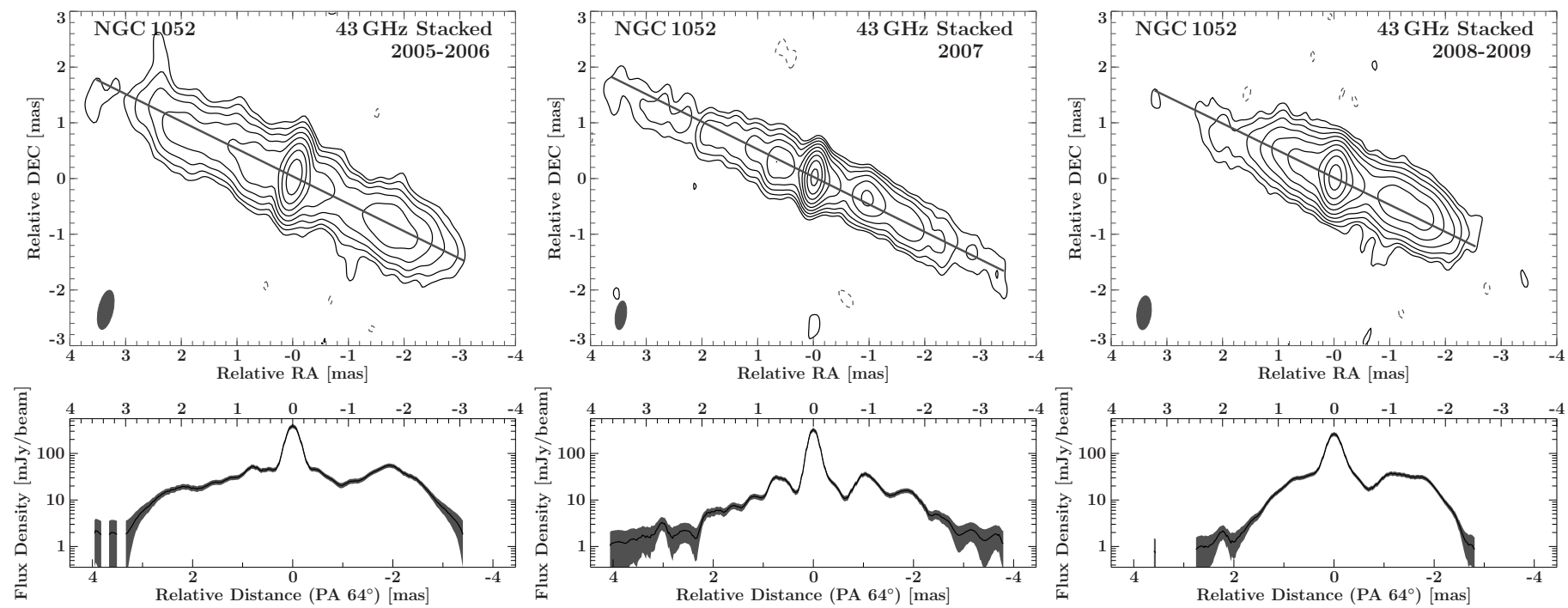

Fig. 10. Stacked images (top panels) with the flux densities along the PA of the jet (bottom panels) for the observations from 2005 to 2006 (left panels), 2007 (middle panels), and 2008-2009 (right panels). The cut along the PA is indicated in the top panels as a line along the jet axis. The common beam for the stacked maps is shown in the bottom left corner of the top panels. For the stacked images the maps have been summed up and divided by the number of images. The contours start at 3 times the noise level and increase logarithmically by factors of 2 .

the surrounding disk that is accreted by the black hole. Assuming there are several small-scale magnetic field loops with alternating polarity (Parfrey et al. 2015; Contopoulos et al. 2018).

For example if we assume a magnetic field configuration in the disk consisting of four magnetic field loops: two loops in the northern hemisphere of the disk and two in the southern hemisphere with alternating polarity. During the accretion of the innermost loops, the magnetic field opens up because of the differential rotation and leads to a poloidal magnetic field with different polarity in the northern and southern hemisphere (see, e.g., Fig. 1 in Parfrey et al. 2015).

If we further assume that the polarity in the northern jet is different from the accreted polarity in the northern hemisphere, the ongoing accretion pushes magnetic field lines of different polarity closer and closer together. This leads to a reconnection event and disrupts or turns off the northern jet for some time until it is established again. On the other hand there is no difference in the polarity in the southern hemisphere, i.e., the southern jet and the accreted loop have the same polarity. Therefore no reconnection event occurs that disturbs the jet. The situation is flipped in the accretion of the next magnetic loop pair, i.e., the northern jet is unperturbed while the southern jet is disrupted. Within such a scenario asymmetric jets with varying outflow can be produced.

A detailed study of the magnetic field in the region of the central engine in NGC 1052 could shed light on whether this could be an explanation for the observed asymmetry. Until now, it was only possible to derive estimates on the mean magnetic field in the central region. Our recent study of NGC 1052 observed with the Global millimeter VLBI array (GMVA) at $86 \mathrm{GHz}$ (Baczko et al. 2016a) sets boundaries to the magnetic field at $1 R_{\mathrm{S}}$ as 200 Gauss $<B<8 \times 10^{4}$ Gauss. However, we do not have linear polarization data that would allow us to infer the geometry of the magnetic field in NGC 1052. Future millimetreVLBI observations may be able to trace changes in the magnetic field of NGC 1052.

Even without detailed knowledge of the magnetic field geometry in NGC 1052 our results favor an asymmetric jet production. Subtracting the influence of the absorbing torus we expect higher flux density values for the western jet close to the central engine as compared to the eastern jet. On the other hand there is a clear trend toward higher velocities in the eastern jet. These observations fit very well into a picture in which the western jet carries a larger internal energy and/or magnetic flux than the eastern jet, thus becoming brighter at these scales. Simultaneous multifrequency observations will allow us to study in detail absorption mechanisms, external and internal, along both jets, thus probing the local magnetic field distribution.

\section{Conclusions}

In this work we present a detailed VLBI analysis of NGC 1052 involving 29 epochs from 2005 to 2009 at $43 \mathrm{GHz}$.

1. In early 2007 the morphology of the source changes from symmetric to asymmetric with the western jet becoming brighter. Further the flux density ratio of the western to eastern jets varies between $R=1.1$ and $R=2.5$.

2. A kinematic analysis of the evolution of the various jet components results in mean apparent jet velocities of $\beta_{\mathrm{wj}}=$ $0.343 \pm 0.037$ and $\beta_{\mathrm{ej}}=0.529 \pm 0.038$ for the western and eastern jets, respectively. Compared to previous estimates at lower frequencies (Vermeulen et al. 2003; Lister et al. 2013) the apparent jet speeds derived in this work are faster, especially for the western jet.

3. Besides the extended jet emission, one central bright emission region is present in all observed epochs. Based on the kinematic analysis this central feature is likely the dynamic center of the system.

4. Combining the flux density evolution and kinematics, we explored the parameter space of the intrinsic velocity and viewing angle. We did not find consistent parameters for all observations, which points toward asymmetric jet production.

5. We analyzed the spectral properties of the source morphology between $22 \mathrm{GHz}$ and $43 \mathrm{GHz}$. It reveals an optically thick region to the west of the brightest jet feature at $22 \mathrm{GHz}$ with a diameter of around 1.5 mas $(0.15 \mathrm{pc})$. The absorption seems to be caused by a torus around the central engine which is perpendicular to the twin-jet system. Both jets are optically thin with spectral indices $\alpha \leq-1$.

6. We fit power laws to the distribution of brightness temperatures and jet diameter as a function of the separation from 
the center. The expansion is similar for both jets, leading to a well-collimated structure, which evolves starting at around 1 mas, with a power-law index of $d=0.65$.

7. The brightness temperature values in the western jet drop at 1 mas separation to the center. This is well be explained by a reduction of the flux density owing to absorption in the torus.

8. We propose a faster inner layer that is fed from a region closer to the central engine, as observed at $43 \mathrm{GHz}$, and a slower moving outer layer, which has particles that come further outward from the accretion disk, as observed at lower frequencies.

9. These observations fit very well into a picture in which the western jet carries a larger internal energy and/or magnetic flux than the eastern jet, thus becoming brighter at these scales.

Further studies at $43 \mathrm{GHz}$ are needed to test the observed asymmetry on longer timescales. As an observation at $86 \mathrm{GHz}$ resulted in a similar morphology (Baczko et al. 2016b), simultaneous observations up to the highest achievable radio frequencies would allow us to perform the core-shift analysis and spectral index measurements up to even higher frequencies. This will result in a more complete picture of the twin-jet system in NGC 1052 and would give us the chance to test current jet formation models.

Acknowledgements. M.P. acknowledges partial support from the Spanish MICINN grant AYA-2013-48226-C03-02-P and the Generalitat Valenciana grant PROMETEOII/2014/069. R.S. gratefully acknowledge support from the European Research Council under the European Union's Seventh Framework Programme (FP/2007-2013)/ERC Advanced Grant RADIOLIFE-320745 CMF is supported by the ERC Synergy Grant "BlackHoleCam - Imaging the Even Horizon of Black Holes" (Grant 610058). This research has made use of the software package isis (Houck \& Denicola 2000) and a collection of Isis scripts provided by the Dr. Karl Remeis Observatory, Bamberg, Germany at http: //www.sternwarte.uni-erlangen.de/isis/, as well as an IsIs-DIFMAP fitting setup by C. Grossberger (Grossberger 2014). This research has made use of SciPy (Jones et al. 2001) and the ODR package, based on Boggs \& Rogers (1990). This research has made use of the NASA/IPAC Extragalactic Database (NED) which is operated by the Jet Propulsion Laboratory, California Institute of Technology, under contract with the National Aeronautics and Space Administration. This research has made use of data obtained with the Very Long Baseline Array (VLBA). The VLBA is an instrument of the National Radio Astronomy Observatory, a facility of the National Science Foundation operated under cooperative agreement by Associated Universities, Inc.

\section{References}

Baczko, A.-K., Schulz, R., Kadler, M., et al. 2016a, A\&A, 593, A47

Baczko, A.-K., Schulz, R., Ros, E., et al. 2016b, Galaxies, 4, 48

Bardeen, J. M., \& Petterson, J. A. 1975, ApJ, 195, L65

Blandford, R. D., \& Königl, A. 1979, ApJ, 232, 34

Böck, M. 2012, PhD Thesis, Friedrich-Alexander-Universität ErlangenNürnberg, Germany

Boggs, P. T., \& Rogers, J. E. 1990, in Statistical Analysis of Measurement Error Models and Applications: Proceedings of the AMS-IMS-SIAM Joint Summer Research Conference held June 10-16, 1989, eds. P. Brown, W. Fuller, A. Society, I. Statistics, \& S. Mathematics (American Mathematical Society), Contemporary Mathematics Series, 112, 186
Bowman, M., Leahy, J. P., \& Komissarov, S. S. 1996, MNRAS, 279, 899

Britzen, S., Qian, S.-J., Steffen, W., et al. 2017, A\&A, 602, A29

Caproni, A., Mosquera Cuesta, H. J., \& Abraham, Z. 2004, ApJ, 616, L99

Claussen, M. J., Diamond, P. J., Braatz, J. A., Wilson, A. S., \& Henkel, C. 1998, ApJ, 500, L129

Contopoulos, I., Nathanail, A., Sạdowski, A., Kazanas, D., \& Narayan, R. 2018, MNRAS, 473, 721

Fendt, C., \& Sheikhnezami, S. 2013, ApJ, 774, 12

Fomalont, E. B. 1999, in Synthesis Imaging in Radio Astronomy II, eds. G. B. Taylor, C. L. Carilli, \& R. A. Perley, ASP Conf. Ser., 180, 301

Fosbury, R. A. E., Mebold, U., Goss, W. M., \& Dopita, M. A. 1978, MNRAS, 183,549

Fromm, C. M., Ros, E., Perucho, M., et al. 2013, A\&A, 557, A105

Fromm, C. M., Perucho, M., Porth, O., et al. 2018, A\&A, 609, A80

Grossberger, C. 2014, PhD Thesis, Friedrich-Alexander-Universität ErlangenNürnberg, Germany

Ho, L. C., Filippenko, A. V., \& Sargent, W. L. W. 1997, ApJS, 112, 315

Houck, J. C., \& Denicola, L. A. 2000, in Astronomical Data Analysis Software and Systems IX, eds. N. Manset, C. Veillet, \& D. Crabtree, ASP Conf. Ser., 216, 591

Jensen, J. B., Tonry, J. L., Barris, B. J., et al. 2003, ApJ, 583, 712

Jones, E., Oliphant, T., Peterson, P., et al. 2001, SciPy: Open Source Scientific Tools for Python

Kameno, S., Sawada-Satoh, S., Inoue, M., Shen, Z.-Q., \& Wajima, K. 2001, PASJ, 53, 169

Kadler, M., Kerp, J., Ros, E., et al. 2004a, A\&A, 420, 467

Kadler, M., Ros, E., Lobanov, A. P., Falcke, H., \& Zensus, J. A. 2004b, A\&A, 426, 481

Kameno, S., Nakai, N., Sawada-Satoh, S., Sato, N., \& Haba, A. 2005, ApJ, 620, 145

Kellermann, K. I., Vermeulen, R. C., Cohen, M. H., \& Zensus, J. A. 1999, BAAS, 31,856

Königl, A. 1981, ApJ, 243, 700

Lister, M. L., Cohen, M. H., Homan, D. C., et al. 2009, AJ, 138, 1874

Lister, M. L., Aller, M. F., Aller, H. D., et al. 2013, AJ, 146, 120

Liu, S., \& Melia, F. 2002, ApJ, 573, L23

Machalski, J., Jamrozy, M., Stawarz, Ł., \& Weżgowiec, M. 2016, A\&A, 595, A46

Margon, B., \& Anderson, S. F. 1989, ApJ, 347, 448

Martí-Vidal, I., Marcaide, J. M., Alberdi, A., et al. 2011, A\&A, 533, A111

Matveyenko, L. I., \& Sivakon', S. S. 2013, Astron. Lett., 39, 481

Mayall, N. U. 1939, PASP, 51, 282

Mohan, P., An, T., Frey, S., et al. 2016, MNRAS, 463, 1812

Perley, R. A., \& Meisenheimer, K. 2017, A\&A, 601, A35

Parfrey, K., Giannios, D., \& Beloborodov, A. M. 2015, MNRAS, 446, L61

Perucho, M., Martí, J. M., Laing, R. A., \& Hardee, P. E. 2014, MNRAS, 441, 1488

Pierce, M., Brodie, J. P., Forbes, D. A., et al. 2005, MNRAS, 358, 419

Pushkarev, A. B., Kovalev, Y. Y., Lister, M. L., \& Savolainen, T. 2017, MNRAS, 468, 4992

Qian, S. J., Britzen, S., Witzel, A., Krichbaum, T. P., \& Gan, H. Q. 2017, A\&A, 604, A90

Rozgonyi, K., \& Frey, S. 2016, Galaxies, 4, 10

Rubinur, K., Das, M., Kharb, P., \& Honey, M. 2017, MNRAS, 465, 4772

Sawada-Satoh, S., Kameno, S., Nakamura, K., et al. 2008, ApJ, 680, 191

Shepherd, M. C., Pearson, T. J., \& Taylor, G. B. 1994, BAAS, 26, 987

Tully, R. B., Courtois, H. M., Dolphin, A. E., et al. 2013, AJ, 146, 86

van Gorkom, J. H., Knapp, G. R., Raimond, E., Faber, S. M., \& Gallagher, J. S. 1986, AJ, 91, 791

Vermeulen, R. C., Ros, E., Kellermann, K. I., et al. 2003, A\&A, 401, 113

Woo, J.-H., \& Urry, C. M. 2002, ApJ, 579, 530 


\section{Appendix A: Additional tables and figures}

Table A.1. Values of reduce chi square from Difmap for Gaussian model fitting.

\begin{tabular}{lclc}
\hline \hline Epoch & $\chi^{2}$ & Epoch & $\chi^{2}$ \\
\hline BR099A & 1.0642407 & BR120E & 1.0231979 \\
BR099C & 0.9321564 & BR120F & 0.6463079 \\
BR099D & 1.0673188 & BR120G & 1.2473867 \\
BR099E & 1.0715107 & BR120H & 0.8063296 \\
BR099F & 0.9859955 & BR120I & 1.0824018 \\
BR099G & 1.1014646 & BR130A & 1.0717157 \\
BR099H & 1.0176922 & BR130B & 1.0158143 \\
BR099I & 0.9272598 & BR130C & 0.9345456 \\
BR119A & 0.9957912 & BR130D & 0.9786728 \\
BR119B & 0.9560460 & BR130E & 0.9293561 \\
BR120A & 0.8111044 & BR130F & 0.8472571 \\
BR120B & 0.8067314 & BR130G & 0.7743667 \\
BR120C & 0.9757917 & BR130H & 0.9449142 \\
BR120D & 0.9645245 & BR130I & 0.9357334 \\
\hline
\end{tabular}

Table A.2. Parameters for all components of epochs BR099A, BR099C, BR099D, BR099E, and BR099F (from top to bottom).

\begin{tabular}{|c|c|c|c|c|c|c|}
\hline Component & $S_{\text {tot }}(\mathrm{Jy})$ & Distance (mas) & $\mathrm{PA}\left({ }^{\circ}\right)$ & Major (mas) & Ratio & $\log T_{\mathrm{b}}(\mathrm{K})$ \\
\hline wj1 & 0.07 & -2.36 & - & 0.36 & 1.00 & 8.56 \\
\hline wj2 & 0.09 & -1.81 & - & 0.28 & 1.00 & 8.89 \\
\hline wj4 & 0.08 & -1.16 & - & 0.21 & 1.00 & 9.07 \\
\hline- & 0.05 & -0.61 & 23.84 & 0.50 & 0.27 & 8.73 \\
\hline Core & 0.38 & 0.00 & 43.83 & 0.15 & 0.00 & - \\
\hline ej3 & 0.08 & 0.59 & - & 0.40 & 1.00 & 8.50 \\
\hline ej2 & 0.07 & 1.12 & - & 0.30 & 1.00 & 8.68 \\
\hline ej1 & 0.04 & 1.66 & - & 0.33 & 1.00 & 8.38 \\
\hline- & 0.06 & 2.28 & 40.86 & 0.65 & 0.58 & 8.24 \\
\hline wj1 & 0.05 & -2.80 & - & 0.47 & 1.00 & 8.16 \\
\hline wj2 & 0.12 & -2.18 & - & 0.34 & 1.00 & 8.85 \\
\hline wj3 & 0.08 & -1.79 & - & 0.15 & 1.00 & 9.36 \\
\hline wj4 & 0.09 & -1.41 & - & 0.36 & 1.00 & 8.68 \\
\hline wj5 & 0.11 & -0.47 & - & 0.30 & 1.00 & 8.92 \\
\hline Core & 0.43 & 0.00 & 5.50 & 0.13 & 0.39 & 10.63 \\
\hline ej4 & 0.07 & 0.48 & - & 0.07 & 1.00 & 9.96 \\
\hline ej3 & 0.15 & 0.82 & - & 0.28 & 1.00 & 9.10 \\
\hline ej2 & 0.05 & 1.53 & - & 0.50 & 1.00 & 8.12 \\
\hline ej1 & 0.03 & 2.19 & - & 0.18 & 1.00 & 8.71 \\
\hline- & 0.03 & 2.58 & - & 0.22 & 1.00 & 8.58 \\
\hline wj1 & 0.02 & -3.13 & - & 0.21 & 1.00 & 8.38 \\
\hline wj2 & 0.08 & -2.40 & - & 0.39 & 1.00 & 8.53 \\
\hline wj3 & 0.08 & -1.92 & - & 0.23 & 1.00 & 9.02 \\
\hline wj4 & 0.06 & -1.49 & - & 0.32 & 1.00 & 8.62 \\
\hline wj5 & 0.05 & -0.78 & - & 0.36 & 1.00 & 8.46 \\
\hline wj6 & 0.09 & -0.31 & - & 0.31 & 1.00 & 8.82 \\
\hline Core & 0.31 & 0.00 & 18.66 & 0.10 & 0.00 & - \\
\hline ej5 & 0.12 & 0.27 & - & 0.41 & 1.00 & 8.66 \\
\hline ej4 & 0.08 & 0.78 & - & 0.23 & 1.00 & 9.04 \\
\hline ej3 & 0.06 & 1.24 & - & 0.37 & 1.00 & 8.45 \\
\hline ej2 & 0.05 & 1.87 & - & 0.52 & 1.00 & 8.04 \\
\hline ej1 & 0.05 & 2.50 & - & 0.44 & 1.00 & 8.23 \\
\hline wj1 & 0.02 & -3.23 & - & 0.29 & 1.00 & 8.23 \\
\hline$w \mathbf{j} 2$ & 0.09 & -2.51 & - & 0.32 & 1.00 & 8.77 \\
\hline wj3 & 0.16 & -1.99 & - & 0.29 & 1.00 & 9.11 \\
\hline wj4 & 0.09 & -1.57 & - & 0.31 & 1.00 & 8.82 \\
\hline wj5 & 0.07 & -0.82 & - & 0.24 & 1.00 & 8.87 \\
\hline wj6 & 0.19 & -0.34 & - & 0.35 & 1.00 & 9.02 \\
\hline Core & 0.48 & 0.00 & 12.03 & 0.18 & 0.00 & - \\
\hline ej5 & 0.15 & 0.31 & - & 0.37 & 1.00 & 8.87 \\
\hline ej4 & 0.14 & 0.81 & - & 0.27 & 1.00 & 9.11 \\
\hline ej3 & 0.09 & 1.35 & - & 0.41 & 1.00 & 8.54 \\
\hline ej2 & 0.08 & 2.07 & - & 0.43 & 1.00 & 8.42 \\
\hline ej1 & 0.04 & 2.57 & - & 0.29 & 1.00 & 8.47 \\
\hline wj2 & 0.08 & -2.67 & - & 0.47 & 1.00 & 8.37 \\
\hline wj3 & 0.11 & -2.07 & - & 0.32 & 1.00 & 8.85 \\
\hline wj4 & 0.06 & -1.59 & - & 0.18 & 1.00 & 9.07 \\
\hline wj5 & 0.06 & -1.04 & - & 0.23 & 1.00 & 8.91 \\
\hline wj6 & 0.10 & -0.50 & - & 0.15 & 1.00 & 9.51 \\
\hline Core & 0.53 & 0.00 & - & 0.08 & 1.00 & 10.74 \\
\hline ej5 & 0.14 & 0.58 & - & 0.42 & 1.00 & 8.72 \\
\hline ej4 & 0.08 & 1.19 & - & 0.24 & 1.00 & 8.95 \\
\hline ej3 & 0.07 & 1.74 & - & 0.32 & 1.00 & 8.66 \\
\hline ej2 & 0.07 & 2.32 & - & 0.43 & 1.00 & 8.38 \\
\hline ej1 & 0.04 & 2.92 & - & 0.37 & 1.00 & 8.34 \\
\hline
\end{tabular}



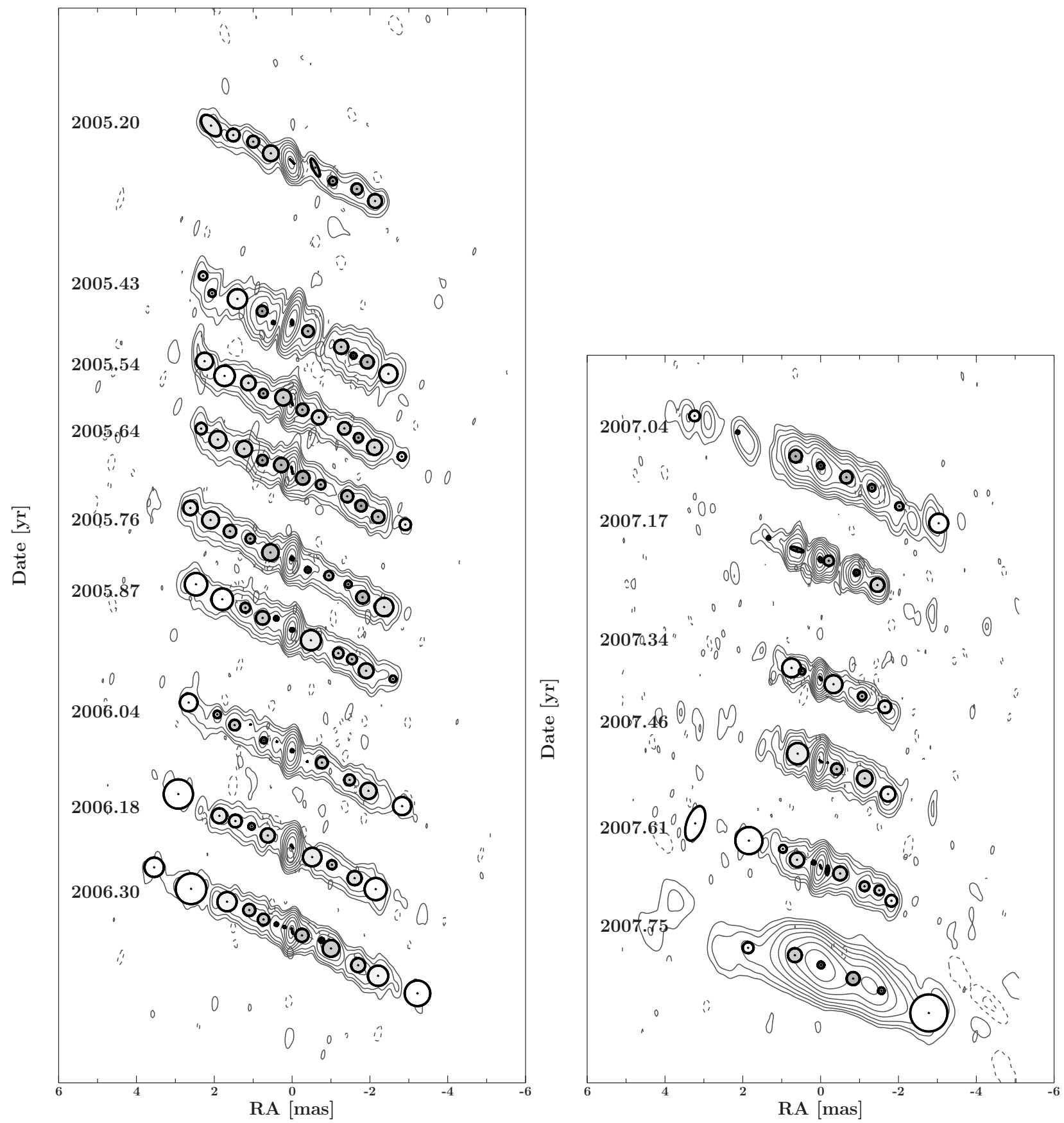

Fig. A.1. cLEan contour maps with overplotted model components for the first and second block at $43 \mathrm{GHz}$. The date of observation is printed left of each image. The brightness temperatures of the components are represented by a filling factor; brighter components correspond to lower brightness temperatures. The contours start at 3 times the noise level and increase logarithmically by factors of 2 . 
Table A.3. Parameters for all components of epoch BR099G, BR099I, BR119A, BR119B, and BR120A (from top to bottom).

\begin{tabular}{|c|c|c|c|c|c|c|}
\hline Component & $S_{\text {tot }}(\mathrm{Jy})$ & Distance (mas) & $\mathrm{PA}\left({ }^{\circ}\right)$ & Major (mas) & Ratio & $\log T_{\mathrm{b}}(\mathrm{K})$ \\
\hline wj2 & 0.02 & -2.88 & - & 0.17 & 1.00 & 8.61 \\
\hline wj3 & 0.07 & -2.17 & - & 0.38 & 1.00 & 8.52 \\
\hline wj4 & 0.05 & -1.71 & - & 0.25 & 1.00 & 8.71 \\
\hline wj5 & 0.05 & -1.33 & - & 0.27 & 1.00 & 8.64 \\
\hline wj6 & 0.09 & -0.55 & - & 0.52 & 1.00 & 8.35 \\
\hline Core & 0.30 & 0.00 & - & 0.09 & 1.00 & 10.40 \\
\hline ej6 & 0.04 & 0.50 & - & 0.11 & 1.00 & 9.37 \\
\hline ej5 & 0.08 & 0.82 & - & 0.35 & 1.00 & 8.64 \\
\hline ej4 & 0.05 & 1.33 & - & 0.25 & 1.00 & 8.76 \\
\hline ej3 & 0.05 & 1.96 & - & 0.55 & 1.00 & 8.03 \\
\hline ej2 & 0.04 & 2.73 & - & 0.58 & 1.00 & 7.87 \\
\hline wj2 & 0.02 & -3.16 & - & 0.47 & 1.00 & 7.88 \\
\hline wj3 & 0.07 & -2.22 & - & 0.42 & 1.00 & 8.41 \\
\hline wj5 & 0.06 & -1.66 & - & 0.28 & 1.00 & 8.70 \\
\hline wj6 & 0.05 & -0.83 & - & 0.30 & 1.00 & 8.62 \\
\hline wj7 & 0.03 & -0.48 & - & 0.00 & 1.00 & - \\
\hline Core & 0.36 & 0.00 & - & 0.07 & 1.00 & 10.68 \\
\hline ej7 & 0.04 & 0.46 & - & 0.00 & 1.00 & - \\
\hline ej6 & 0.05 & 0.77 & - & 0.16 & 1.00 & 9.15 \\
\hline ej5 & 0.02 & 1.26 & - & 0.00 & 1.00 & 20.05 \\
\hline ej4 & 0.05 & 1.62 & - & 0.27 & 1.00 & 8.64 \\
\hline ej3 & 0.02 & 2.14 & - & 0.19 & 1.00 & 8.63 \\
\hline ej2 & 0.03 & 2.93 & - & 0.45 & 1.00 & 7.99 \\
\hline wj3 & 0.05 & -2.41 & - & 0.57 & 1.00 & 8.03 \\
\hline wj5 & 0.06 & -1.80 & - & 0.36 & 1.00 & 8.47 \\
\hline wj6 & 0.05 & -1.12 & - & 0.23 & 1.00 & 8.81 \\
\hline wj7 & 0.06 & -0.58 & - & 0.48 & 1.00 & 8.24 \\
\hline Core & 0.44 & 0.00 & 40.59 & 0.09 & 0.00 & 18.64 \\
\hline ej7 & 0.06 & 0.69 & - & 0.36 & 1.00 & 8.48 \\
\hline ej6 & 0.04 & 1.16 & - & 0.17 & 1.00 & 8.94 \\
\hline ej5 & 0.03 & 1.60 & - & 0.33 & 1.00 & 8.29 \\
\hline ej4 & 0.04 & 2.03 & - & 0.38 & 1.00 & 8.24 \\
\hline ej2 & 0.04 & 3.22 & - & 0.75 & 1.00 & 7.65 \\
\hline$w j 2$ & 0.02 & -3.60 & - & 0.66 & 1.00 & 7.44 \\
\hline wj3 & 0.06 & -2.49 & - & 0.52 & 1.00 & 8.14 \\
\hline wj5 & 0.06 & -1.91 & - & 0.36 & 1.00 & 8.50 \\
\hline wj6 & 0.12 & -1.09 & - & 0.43 & 1.00 & 8.64 \\
\hline wj7 & 0.03 & -0.80 & - & 0.13 & 1.00 & 9.02 \\
\hline- & 0.09 & -0.28 & - & 0.34 & 1.00 & 8.71 \\
\hline Core & 0.42 & 0.00 & 12.03 & 0.14 & 0.00 & - \\
\hline- & 0.04 & 0.22 & - & 0.05 & 1.00 & 10.03 \\
\hline- & 0.04 & 0.44 & - & 0.08 & 1.00 & 9.57 \\
\hline ej7 & 0.05 & 0.79 & - & 0.29 & 1.00 & 8.62 \\
\hline ej6 & 0.06 & 1.23 & - & 0.31 & 1.00 & 8.60 \\
\hline ej5 & 0.06 & 1.83 & - & 0.49 & 1.00 & 8.23 \\
\hline ej3 & 0.06 & 2.82 & - & 0.79 & 1.00 & 7.79 \\
\hline ej1 & 0.02 & 3.91 & - & 0.50 & 1.00 & 7.68 \\
\hline- & 0.02 & -3.37 & - & 0.49 & 1.00 & 7.75 \\
\hline- & 0.03 & -2.27 & - & 0.21 & 1.00 & 8.72 \\
\hline wj8 & 0.09 & -1.43 & - & 0.18 & 1.00 & 9.25 \\
\hline wj9 & 0.13 & -0.72 & - & 0.32 & 1.00 & 8.93 \\
\hline Core & 0.32 & 0.00 & - & 0.18 & 1.00 & 9.81 \\
\hline- & 0.14 & 0.69 & - & 0.32 & 1.00 & 8.95 \\
\hline- & 0.02 & 2.30 & - & 0.08 & 1.00 & 9.34 \\
\hline- & 0.02 & 3.48 & - & 0.27 & 1.00 & 8.14 \\
\hline
\end{tabular}

Notes. Delta-like components with an unphysical high brightness temperature larger than $10^{18} \mathrm{~K}$ have been excluded for each analysis based on brightness temperatures.

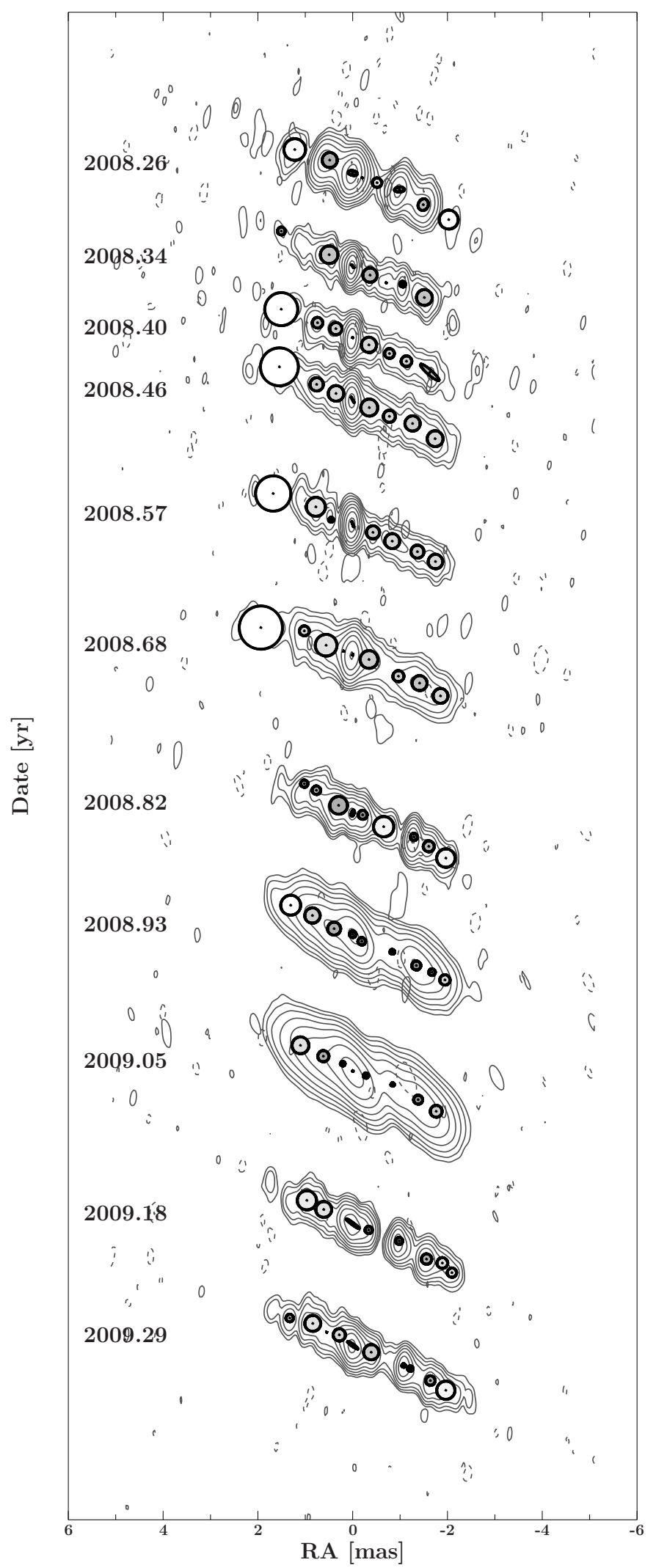

Fig. A.2. CLEAn contour maps with overplotted model for the third block at $43 \mathrm{GHz}$. The date of observation is printed left of each image. The brightness temperatures of the components are represented by a filling factor; brighter components correspond to lower brightness temperatures. The contours start at 3 times the noise level and increase logarithmically by factors of 2 . 

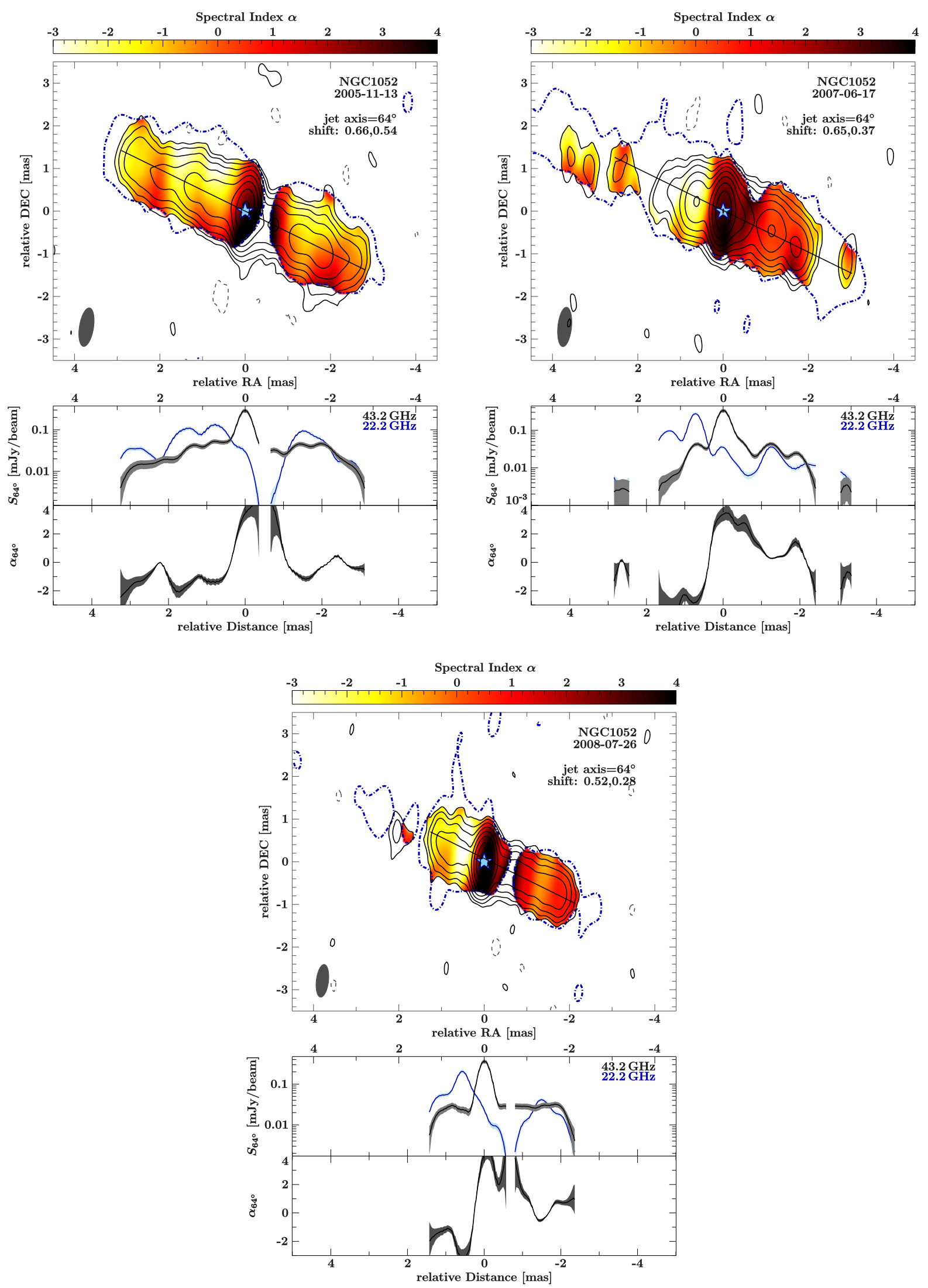

Fig. A.3. Spectral index maps between $22 \mathrm{GHz}$ (dot-dashed blue contour) and $43 \mathrm{GHz}$ (solid black contours) for three selected observations after alignment based on optically thin features (compare Fig. 2). The restoring beam for each epoch is plotted in the lower left corner. A cut along the direction of the jets $\left(64^{\circ}\right)$ results in the bottom panels, showing the spectral index (bottom panels) and the flux density (middle panel) along the jet axis. The blue star indicates the assumed location of the dynamical center. 
Table A.4. Parameters for all components of epoch BR120B, BR120C, BR120D, BR120E, BR120F, BR120H, and BR120I (from top to bottom).

\begin{tabular}{|c|c|c|c|c|c|c|}
\hline Component & $S_{\text {tot }}(\mathrm{Jy})$ & Distance (mas) & $\mathrm{PA}\left(\left(^{\circ}\right)\right.$ & Major (mas) & Ratio & $\log T_{\mathrm{b}}(\mathrm{K})$ \\
\hline wj8 & 0.05 & -1.60 & - & 0.35 & 1.00 & 8.48 \\
\hline wj9 & 0.11 & -0.98 & - & 0.15 & 1.00 & 9.55 \\
\hline wj10 & 0.07 & -0.21 & - & 0.26 & 1.00 & 8.87 \\
\hline core & 0.28 & 0.00 & 26.09 & 0.14 & 0.62 & 10.21 \\
\hline ej9 & 0.14 & 0.66 & 73.04 & 0.35 & 0.27 & 9.44 \\
\hline ej8 & 0.01 & 1.45 & - & 0.08 & 1.00 & 8.93 \\
\hline wj8 & 0.03 & -1.80 & - & 0.32 & 1.00 & 8.27 \\
\hline wj9 & 0.06 & -1.16 & - & 0.22 & 1.00 & 8.93 \\
\hline wj10 & 0.07 & -0.36 & - & 0.46 & 1.00 & 8.32 \\
\hline core & 0.27 & 0.00 & 28.12 & 0.10 & 0.28 & 10.83 \\
\hline ej10 & 0.02 & 0.51 & - & 0.16 & 1.00 & 8.76 \\
\hline ej9 & 0.05 & 0.80 & - & 0.48 & 1.00 & 8.12 \\
\hline wj8 & 0.04 & -1.93 & - & 0.38 & 1.00 & 8.23 \\
\hline wj9 & 0.08 & -1.22 & - & 0.40 & 1.00 & 8.51 \\
\hline wj10 & 0.07 & -0.46 & - & 0.29 & 1.00 & 8.71 \\
\hline- & 0.03 & -0.18 & - & 0.00 & 1.00 & 20.59 \\
\hline core & 0.33 & 0.00 & 49.60 & 0.08 & 0.00 & - \\
\hline ej10 & 0.09 & 0.62 & - & 0.55 & 1.00 & 8.31 \\
\hline wj8 & 0.02 & -2.02 & - & 0.30 & 1.00 & 8.15 \\
\hline- & 0.03 & -1.62 & - & 0.25 & 1.00 & 8.52 \\
\hline wj9 & 0.04 & -1.23 & - & 0.25 & 1.00 & 8.69 \\
\hline wj10 & 0.06 & -0.53 & - & 0.35 & 1.00 & 8.51 \\
\hline- & 0.04 & -0.20 & -4.58 & 0.24 & 0.30 & 9.12 \\
\hline core & 0.27 & 0.00 & 20.67 & 0.09 & 0.00 & - \\
\hline- & 0.02 & 0.21 & - & 0.09 & 1.00 & 9.22 \\
\hline ej10 & 0.05 & 0.63 & - & 0.37 & 1.00 & 8.40 \\
\hline ej9 & 0.01 & 1.07 & - & 0.20 & 1.00 & 8.30 \\
\hline ej8 & 0.01 & 1.96 & - & 0.70 & 1.00 & 7.24 \\
\hline- & 0.01 & 3.41 & -19.34 & 0.93 & 0.48 & 7.32 \\
\hline- & 0.02 & -3.03 & - & 0.95 & 1.00 & 7.11 \\
\hline wj9 & 0.08 & -1.69 & - & 0.17 & 1.00 & 9.28 \\
\hline wj10 & 0.12 & -0.90 & - & 0.34 & 1.00 & 8.85 \\
\hline core & 0.33 & 0.00 & - & 0.17 & 1.00 & 9.88 \\
\hline ej10 & 0.09 & 0.72 & - & 0.36 & 1.00 & 8.65 \\
\hline ej8 & 0.01 & 1.92 & - & 0.28 & 1.00 & 7.95 \\
\hline wj11 & 0.05 & -1.78 & 48.69 & 0.51 & 0.20 & 8.83 \\
\hline wj12 & 0.04 & -1.24 & - & 0.22 & 1.00 & 8.68 \\
\hline wj13 & 0.03 & -0.84 & - & 0.21 & 1.00 & 8.64 \\
\hline wj14 & 0.05 & -0.37 & - & 0.32 & 1.00 & 8.54 \\
\hline core & 0.26 & 0.00 & - & 0.00 & 1.00 & 22.56 \\
\hline ej13 & 0.05 & 0.40 & - & 0.26 & 1.00 & 8.67 \\
\hline ej12 & 0.04 & 0.81 & - & 0.22 & 1.00 & 8.72 \\
\hline ej11 & 0.03 & 1.62 & - & 0.70 & 1.00 & 7.55 \\
\hline
\end{tabular}

Notes. Delta-like components with an unphysical high brightness temperature larger than $10^{18} \mathrm{~K}$ have been excluded for each analysis based on brightness temperatures.
Table A.5. Parameters for all components of epoch BR130A, BR130B, BR130C, BR130D, BR130E, and BR130F (from top to bottom).

\begin{tabular}{|c|c|c|c|c|c|c|}
\hline Component & $S_{\text {tot }}(\mathrm{Jy})$ & Distance (mas) & $\mathrm{PA}\left({ }^{\circ}\right)$ & Major (mas) & Ratio & $\log T_{\mathrm{b}}(\mathrm{K}$ \\
\hline wj11 & 0.10 & -1.65 & - & 0.33 & 1.00 & 8.77 \\
\hline wj12 & 0.06 & -1.12 & - & 0.12 & 1.00 & 9.42 \\
\hline wj13 & 0.02 & -0.78 & - & 0.00 & 1.00 & 21.76 \\
\hline wj14 & 0.10 & -0.41 & - & 0.30 & 1.00 & 8.86 \\
\hline core & 0.29 & 0.00 & 40.50 & 0.08 & 0.00 & - \\
\hline ej12 & 0.12 & 0.55 & - & 0.37 & 1.00 & 8.77 \\
\hline ej11 & 0.02 & 1.68 & - & 0.16 & 1.00 & 8.73 \\
\hline wj11 & 0.06 & -1.92 & - & 0.34 & 1.00 & 8.55 \\
\hline wj12 & 0.07 & -1.36 & - & 0.32 & 1.00 & 8.65 \\
\hline wj13 & 0.03 & -0.85 & - & 0.26 & 1.00 & 8.53 \\
\hline wj14 & 0.08 & -0.39 & - & 0.36 & 1.00 & 8.61 \\
\hline core & 0.28 & 0.00 & 27.61 & 0.13 & 0.00 & - \\
\hline ej13 & 0.06 & 0.38 & - & 0.33 & 1.00 & 8.56 \\
\hline ej12 & 0.05 & 0.83 & - & 0.28 & 1.00 & 8.60 \\
\hline ej11 & 0.02 & 1.70 & - & 0.80 & 1.00 & 7.39 \\
\hline wj11 & 0.05 & -1.92 & - & 0.31 & 1.00 & 8.55 \\
\hline wj12 & 0.05 & -1.48 & - & 0.27 & 1.00 & 8.63 \\
\hline wj13 & 0.04 & -0.91 & - & 0.32 & 1.00 & 8.47 \\
\hline wj14 & 0.05 & -0.46 & - & 0.28 & 1.00 & 8.68 \\
\hline core & 0.37 & 0.00 & 19.85 & 0.12 & 0.00 & - \\
\hline ej13 & 0.03 & 0.46 & - & 0.10 & 1.00 & 9.23 \\
\hline ej12 & 0.07 & 0.86 & - & 0.40 & 1.00 & 8.45 \\
\hline ej11 & 0.02 & 1.80 & - & 0.74 & 1.00 & 7.34 \\
\hline wj11 & 0.05 & -2.05 & - & 0.31 & 1.00 & 8.49 \\
\hline wj12 & 0.08 & -1.53 & - & 0.32 & 1.00 & 8.71 \\
\hline wj13 & 0.04 & -1.07 & - & 0.23 & 1.00 & 8.69 \\
\hline wj14 & 0.09 & -0.36 & - & 0.37 & 1.00 & 8.62 \\
\hline core & 0.31 & 0.00 & -24.93 & 0.02 & 0.00 & - \\
\hline ej14 & 0.02 & 0.20 & - & 0.00 & 1.00 & 21.95 \\
\hline ej13 & 0.08 & 0.60 & - & 0.46 & 1.00 & 8.42 \\
\hline ej12 & 0.02 & 1.14 & - & 0.21 & 1.00 & 8.56 \\
\hline ej11 & 0.03 & 2.02 & - & 0.91 & 1.00 & 7.34 \\
\hline wj11 & 0.03 & -2.18 & - & 0.39 & 1.00 & 8.18 \\
\hline wj12 & 0.05 & -1.75 & - & 0.23 & 1.00 & 8.83 \\
\hline wj13 & 0.14 & -1.39 & - & 0.15 & 1.00 & 9.60 \\
\hline wj14 & 0.05 & -0.71 & - & 0.43 & 1.00 & 8.23 \\
\hline wj15 & 0.13 & -0.21 & - & 0.19 & 1.00 & 9.39 \\
\hline core & 0.09 & 0.00 & -14.80 & 0.14 & 0.42 & 9.85 \\
\hline ej14 & 0.22 & 0.34 & - & 0.37 & 1.00 & 9.01 \\
\hline ej13 & 0.05 & 0.91 & - & 0.18 & 1.00 & 9.02 \\
\hline ej12 & 0.03 & 1.20 & - & 0.15 & 1.00 & 8.92 \\
\hline wj11 & 0.02 & -2.17 & - & 0.22 & 1.00 & 8.50 \\
\hline wj12 & 0.04 & -1.85 & - & 0.14 & 1.00 & 9.17 \\
\hline wj13 & 0.11 & -1.50 & - & 0.19 & 1.00 & 9.30 \\
\hline wj14 & 0.05 & -0.91 & - & 0.10 & 1.00 & 9.54 \\
\hline wj15 & 0.14 & -0.24 & - & 0.16 & 1.00 & 9.56 \\
\hline core & 0.14 & 0.00 & - & 0.14 & 1.00 & 9.68 \\
\hline ej14 & 0.12 & 0.41 & - & 0.28 & 1.00 & 9.03 \\
\hline ej13 & 0.08 & 0.94 & - & 0.32 & 1.00 & 8.70 \\
\hline ej12 & 0.02 & 1.44 & - & 0.42 & 1.00 & 7.82 \\
\hline
\end{tabular}

Notes. Delta-like components with an unphysical high brightness temperature larger than $10^{18} \mathrm{~K}$ have been excluded for each analysis based on brightness temperatures. 
A.-K. Baczko et al.: Asymmetric jet production in NGC 1052

Table A.6. Parameters for all components of epoch BR130G, BR130H, and BR130I (from top to bottom).

\begin{tabular}{ccccccc}
\hline \hline Component & $S_{\text {tot }}(\mathrm{Jy})$ & Distance (mas) & PA $\left(^{\circ}\right)$ & Major (mas) & Ratio & $\log T_{\mathrm{b}}(\mathrm{K})$ \\
\hline wj12 & 0.05 & -1.95 & - & 0.25 & 1.00 & 8.70 \\
wj13 & 0.09 & -1.51 & - & 0.19 & 1.00 & 9.20 \\
wj14 & 0.07 & -0.89 & - & 0.07 & 1.00 & 9.93 \\
wj15 & 0.08 & -0.30 & - & 0.11 & 1.00 & 9.65 \\
core & 0.16 & 0.00 & - & 0.01 & 1.00 & 11.73 \\
ej15 & 0.11 & 0.26 & - & 0.10 & 1.00 & 9.90 \\
ej14 & 0.08 & 0.70 & - & 0.24 & 1.00 & 9.00 \\
ej13 & 0.06 & 1.23 & - & 0.36 & 1.00 & 8.46 \\
\hline wj11 & 0.01 & -2.33 & - & 0.20 & 1.00 & 8.38 \\
wj12 & 0.02 & -2.06 & - & 0.23 & 1.00 & 8.42 \\
wj13 & 0.08 & -1.73 & - & 0.22 & 1.00 & 9.02 \\
wj14 & 0.09 & -1.04 & - & 0.15 & 1.00 & 9.41 \\
wj15 & 0.06 & -0.36 & - & 0.15 & 1.00 & 9.25 \\
core & 0.28 & 0.00 & 53.49 & 0.34 & 0.15 & 10.04 \\
ej14 & 0.05 & 0.68 & - & 0.33 & 1.00 & 8.45 \\
ej13 & 0.05 & 1.09 & - & 0.41 & 1.00 & 8.34 \\
\hline wj12 & 0.04 & -2.18 & - & 0.39 & 1.00 & 8.22 \\
wj13 & 0.06 & -1.80 & - & 0.20 & 1.00 & 8.97 \\
- & 0.02 & -1.31 & - & 0.12 & 1.00 & 9.08 \\
wj14 & 0.07 & -1.16 & - & 0.08 & 1.00 & 9.91 \\
wj15 & 0.07 & -0.42 & - & 0.32 & 1.00 & 8.68 \\
core & 0.28 & 0.00 & 54.02 & 0.26 & 0.19 & 10.15 \\
- & 0.04 & 0.36 & - & 0.26 & 1.00 & 8.56 \\
- & 0.01 & 0.62 & - & 0.00 & 1.00 & 21.90 \\
ej14 & 0.05 & 0.96 & - & 0.34 & 1.00 & 8.45 \\
ej13 & 0.01 & 1.45 & - & 0.16 & 1.00 & 8.57 \\
\hline & & & & & & \\
\hline & & & -15 & \\
\hline
\end{tabular}

Notes. Delta-like components with an unphysical high brightness temperature larger than $10^{18} \mathrm{~K}$ have been excluded for each analysis based on brightness temperatures. 